TRANSACTIONS OF THE

AMERICAN MATHEMATICAL SOCIETY

Volume 364, Number 8, August 2012, Pages 4053-4083

S 0002-9947(2012)05541-3

Article electronically published on March 22, 2012

\title{
SEMICONJUGACIES, PINCHED CANTOR BOUQUETS AND HYPERBOLIC ORBIFOLDS
}

\author{
HELENA MIHALJEVIĆ-BRANDT
}

\begin{abstract}
Let $f: \mathbb{C} \rightarrow \mathbb{C}$ be a transcendental entire map that is subhyperbolic, i.e., the intersection of the Fatou set $\mathcal{F}(f)$ and the postsingular set $P(f)$ is compact and the intersection of the Julia set $\mathcal{J}(f)$ and $P(f)$ is finite. Assume that no asymptotic value of $f$ belongs to $\mathcal{J}(f)$ and that the local degree of $f$ at all points in $\mathcal{J}(f)$ is bounded by some finite constant. We prove that there is a hyperbolic map $g \in\{z \mapsto f(\lambda z): \lambda \in \mathbb{C}\}$ with connected Fatou set such that $f$ and $g$ are semiconjugate on their Julia sets. Furthermore, we show that this semiconjugacy is a conjugacy when restricted to the escaping set $I(g)$ of $g$. In the case where $f$ can be written as a finite composition of maps of finite order, our theorem, together with recent results on Julia sets of hyperbolic maps, implies that $\mathcal{J}(f)$ is a pinched Cantor bouquet, consisting of dynamic rays and their endpoints. Our result also seems to give the first complete description of topological dynamics of an entire transcendental map whose Julia set is the whole complex plane.
\end{abstract}

\section{INTRODUCTION}

It is well known that the Julia set $\mathcal{J}(f)$ of a transcendental entire function $f$ can be the whole complex plane. (Basic definitions and notation are reviewed in Section 2) As far as we know, there is no function with this property for which the topological dynamics has been completely understood. The results in this paper provide such a description for a wide class of examples, including maps such as $z \mapsto \pi \sinh z$. (A description of the combinatorial dynamics of the latter map was previously given by Schleicher in 24.)

In particular, we give an answer to the question of Bergweiler (personal communication) as to whether the escaping set

$$
I(f):=\left\{z \in \mathbb{C}: f^{n}(z) \rightarrow \infty \text { as } n \rightarrow \infty\right\}
$$

of a cosine map $F_{a, b}(z):=a \mathrm{e}^{z}+b \mathrm{e}^{-z}$ with strictly preperiodic critical values is connected: this is not the case; see Corollary 1.4 below. (On the other hand, there are entire maps whose Julia set equals $\mathbb{C}$ but for which the escaping set is connected; see [20, 19.)

In fact, our results are considerably more general. A transcendental entire map $f$ is called subhyperbolic if the intersection of the Fatou set $\mathcal{F}(f)$ and the postsingular set $P(f)$ is compact and the intersection of the Julia set $\mathcal{J}(f)$ and $P(f)$ is finite.

Received by the editors November 27, 2009 and, in revised form, July 5, 2010.

2010 Mathematics Subject Classification. Primary 37F10; Secondary 30D05, 37F30, 37C15, $37 \mathrm{D} 20$.

This work was supported by the Engineering and Physical Sciences Research Council (EPSRC), grant-code: EP/E05285, and was partly supported by the EU Research Training Network Cody.

(C) 2012 American Mathematical Society
Reverts to public domain 28 years from publication 
A subhyperbolic map is called hyperbolic if $\mathcal{J}(f) \cap P(f)=\emptyset$. We are interested in the following class of subhyperbolic functions which includes all hyperbolic maps.

Definition 1.1 (Strongly subhyperbolic maps). A subhyperbolic transcendental entire map $f$ is called strongly subhyperbolic if $\mathcal{J}(f)$ contains no asymptotic values of $f$ and the local degree of $f$ at the points in $\mathcal{J}(f)$ is uniformly bounded.

Note that the map $z \mapsto \pi \sinh z$ mentioned previously is strongly subhyperbolic, since $P(f)=P(f) \cap \mathcal{J}(f)=\{ \pm \pi i, 0\}$ is finite, all critical points are simple and there are no asymptotic values. Our main theorem describes the Julia set of any strongly subhyperbolic entire function as a quotient of the Julia set of a (particularly simple) hyperbolic function in the same parameter space.

Theorem 1.2. Let $f$ be strongly subhyperbolic, and let $\lambda \in \mathbb{C}$ be such that $g(z):=$ $f(\lambda z)$ is hyperbolic with connected Fatou set. Then there exists a continuous surjection $\phi: \mathcal{J}(g) \rightarrow \mathcal{J}(f)$, such that

$$
f(\phi(z))=\phi(g(z))
$$

for all $z \in \mathcal{J}(g)$. Moreover, $\phi$ restricts to a homeomorphism between the escaping sets $I(g)$ and $I(f)$.

Remark. The hypothesis will be automatically satisfied whenever $\lambda$ is sufficiently small. Also, any two maps $g$ and $g^{\prime}$ as in the theorem are quasiconformally conjugate on a neighbourhood of their Julia sets [18], so it is sufficient to prove the theorem for any such map.

As in [18, we say that a hyperbolic function $g$ with connected Fatou set is of disjoint type. For simple families, such as $z \mapsto \lambda \sinh z$, the dynamics of disjoint type functions is well understood. Hence Theorem 1.2 extends this understanding to all strongly subhyperbolic functions in these families. (In Appendix A, we present a detailed description of the topological dynamics of $z \mapsto \pi \sinh$.)

More generally, suppose that $g$ is of disjoint type and has finite order, i.e., $\log \log |g(z)|=\mathrm{O}(\log |z|)$ as $z \rightarrow \infty$, or, more generally, can be written as a finite composition of finite-order maps with bounded singular sets. Then it is known that $\mathcal{J}(g)$ is a Cantor bouquet, i.e., homeomorphic to a straight brush in the sense of [1].

Corollary 1.3. Let $f=f_{1} \circ \cdots \circ f_{n}$ be a strongly subhyperbolic map, where every $f_{i}$ is an entire map with bounded set of singular values and with finite order of growth. Then $\mathcal{J}(f)$ is a pinched Cantor bouquet; that is, the quotient of a Cantor bouquet by a closed equivalence relation defined on its endpoints.

The escaping set of a disjoint type entire function is always disconnected, so we also have the following corollary, settling Bergweiler's question for all strongly subhyperbolic maps.

Corollary 1.4. The escaping set of a strongly subhyperbolic transcendental entire function is disconnected.

Let us comment on the assumption of strong subhyperbolicity. Theorem 1.2 is not true for all subhyperbolic maps: it is known that $E_{1}: z \mapsto \frac{1}{e^{2}} e^{z}$ (disjoint type) and $E_{2}: z \mapsto 2 \pi i e^{z}$ (subhyperbolic) are not topologically conjugate on their escaping sets [17, Proposition 2.1]. (In fact, for exponential maps the escaping set consists of curves to infinity, called dynamic rays $\left[23\right.$. For $E_{1}$, all these rays have a 
landing point in $\mathbb{C}$, while for $E_{2}$ there are uncountably many dynamic rays, each of which accumulates everywhere upon itself.) Rempe asked whether two cosine maps with strictly preperiodic critical values can be conjugate on their escaping sets [17, Question 12.1]. Theorem 1.2 together with the mentioned result in [18] on disjoint type maps gives an affirmative answer to this question.

For cosine maps $F_{a, b}(z)=a \mathrm{e}^{z}+b \mathrm{e}^{-z}$ that are subhyperbolic, Theorem $1.2 \mathrm{im}$ plies that every point in the Julia set is either on a dynamic ray or the landing point of a dynamic ray. This has already been shown by Schleicher 24 when both critical values of $F_{a, b}$ are assumed to be preperiodic. Nonetheless, his results do not explain the topological embedding of the escaping set of such a map in the complex plane. Furthermore, Theorem 1.2 formulated for subhyperbolic cosine maps can be proved in a concise and fairly elementary way, which is why we have included the modifications of our proof for this special case in Section 4.4

For hyperbolic maps, Theorem 1.2 is due to Rempe, and our proof is in the spirit of the ideas presented in [18. However, the attempt to transfer the construction in the hyperbolic case to the setting of strongly subhyperbolic maps fails due to the existence of singular values in the Julia sets. This obstruction is overcome by studying Julia sets as subsets of hyperbolic Riemann orbifolds. These can be thought of as images of the unit disk under branched coverings, for which the set of critical values is discrete and "tame". This yields a description of a Riemann orbifold as a Riemann surface together with a discrete set of ramified points, each of which has finite ramification value. The use of orbifolds in dynamics goes back to Thurston and they have been used with great success by Douady and Hubbard in their work on subhyperbolic rational maps. The following result is not only crucial for the proof of Theorem 1.2 but also interesting in its own right, since it provides us with a global estimate of the hyperbolic metric on certain hyperbolic Riemann orbifolds.

Theorem 1.5. Let $K>1$ and let $z_{j} \rightarrow \infty$ be a sequence of points satisfying $\left|z_{j}\right|<\left|z_{j+1}\right| \leq K\left|z_{j}\right|$. Let $\mathcal{O}$ be the orbifold with $\mathbb{C}$ as the underlying surface and whose ramified points are the points $z_{i}$ with ramification value 2.

Then the density $\rho_{\mathcal{O}}$ of the hyperbolic metric on $\mathcal{O}$ satisfies

$$
\frac{1}{\rho_{\mathcal{O}}(z)} \leq \mathrm{O}(|z|) \quad \text { as } \quad z \rightarrow \infty \text {. }
$$

The orbifold for which the set of ramified points is given by $\{2 k \pi i: k \in \mathbb{Z}\}$ shows that our estimate is best possible (see the proof of Proposition 4.6).

Remark. If we replace the ramified points by punctures, i.e., if we consider the hyperbolic domain $U:=\mathbb{C} \backslash\left\{z_{j}: j \in \mathbb{N}\right\}$ instead of the orbifold $\mathcal{O}$, the same bound for the asymptotic behaviour of the density map near $\infty$ can be obtained using standard estimates of the hyperbolic metric in the twice-punctured plane [18, Lemma 2.1].

It will become clear in Section 3 why strongly subhyperbolic maps are exactly those maps which can be approached with orbifold theory. This still leaves open the question as to what can be said about topological dynamics of maps that are subhyperbolic but not strongly subhyperbolic. We believe that if $\mathcal{J}(f)$ contains an asymptotic value of $f$, then, similar to the case of exponential maps, there is no conjugacy between $f$ and any (suitable) disjoint type map $g$ on their escaping sets; this is work in progress. However, we have no indication of what to expect 
for maps whose Julia sets contain no asymptotic values but sequences of points with unbounded local degree. It would be very interesting to explore this problem, in particular since there are prominent examples of such maps such as Poincaré functions corresponding to certain hyperbolic polynomials. An elaboration of such an example is given in Appendix B.

Structure of the article. In Section 3 we develop the concept of orbifolds $d y$ namically associated to a strongly subhyperbolic map $f$. The main consequence is that we obtain a hyperbolic orbifold $\mathcal{O}_{f}$ such that $f$ is expanding with respect to the corresponding hyperbolic metric. Later, in Section 4 we prove that the expansion of $f$ is actually uniform. The key for this will be (the proof of) Theorem 1.5. Finally, Section 5 addresses the construction of the semiconjugacy itself.

\section{Preliminaries}

If not stated differently, we will assume throughout this article that the maps considered are transcendental entire. We denote the complex plane by $\mathbb{C}$, the Riemann sphere by $\widehat{\mathbb{C}}:=\mathbb{C} \cup\{\infty\}$, and the punctured plane by $\mathbb{C}^{*}:=\mathbb{C} \backslash\{0\}$. We write $\mathbb{D}$ for the unit disk and $\mathbb{H}$ for the upper half-plane. The Euclidean neighbourhood of a set $A \subset \mathbb{C}$ will be denoted by $D_{\varepsilon}(A)$, so in particular, if $c$ is a point in $\mathbb{C}$, then $D_{r}(c)$ means the Euclidean disk centred at $c$ with radius $r$. If not stated differently, the boundary $\partial A$ and the closure $\bar{A}$ of a set $A \subset \mathbb{C}$ are always understood to be taken relative to the complex plane. The Euclidean distance between two sets $A, B \subset \mathbb{C}$ will be denoted by $\operatorname{dist}(A, B)$. For $A \subset B \subset \mathbb{C}$ we will write $A \Subset B$ if $A$ is compactly contained in $B$, i.e., if $\bar{A}$ is a compact subset of $B$. For a sequence $n_{i}$ of natural numbers we write $\operatorname{lcm}\left\{n_{i}\right\}$ for their least common multiple.

2.1. Background on holomorphic dynamics. The Fatou set of a map $f$ is the set of all points in $\mathbb{C}$ that have a neighbourhood in which the iterates $\left\{f^{n}\right\}$ form a normal family in the sense of Montel. Its complement $\mathcal{J}(f):=\mathbb{C} \backslash \mathcal{F}(f)$ is called the Julia set of $f$. Recall that the escaping set of $f$ is given by

$$
I(f):=\left\{z \in \mathbb{C}: f^{n}(z) \rightarrow \infty \text { as } n \rightarrow \infty\right\} .
$$

We say that a point $z \in \mathbb{C}$ is a periodic point of $f$ if there exists an integer $n \geq 1$ such that $f^{n}(z)=z$. The smallest $n$ with this property is called the period of $z$. A periodic point of period one is called a fixed point. We call a point $z$ preperiodic under $f$ if some image $f^{n}(z), n \geq 1$, of $z$ is periodic. Note that every periodic point is also preperiodic. To avoid confusion, we say that a point $z$ is strictly preperiodic if it is preperiodic but not periodic. Let $z$ be a periodic point of $f$ of period $n$. We call $\mu(z):=\left(f^{n}\right)^{\prime}(z)$ the multiplier of $z$. A periodic point $z$ is called attracting if $0 \leq|\mu(z)|<1$, indifferent if $|\mu(z)|=1$ and repelling if $|\mu(z)|>1$. Since the multiplier of an indifferent periodic point is of the form $\mathrm{e}^{2 \pi i t}$ with $0 \leq t<1$, we can distinguish between rationally and irrationally indifferent points, according to whether or not $t$ is rational. The set of all points whose orbits converge to an attracting periodic cycle is called the attracting basin of this cycle.

We denote the set of all critical points of $f$ by Crit $(f)$, the set of all critical values by $C(f)=f(\operatorname{Crit}(f))$ and the set of all (finite) asymptotic values by $A(f)$. The set of singular values of $f$, denoted by $S(f)$, is the smallest closed set such 
that $f: \mathbb{C} \backslash f^{-1}(S(f)) \rightarrow \mathbb{C} \backslash S(f)$ is a covering map. It is well known that $S(f):=\overline{C(f) \cup A(f)}$. Finally, we denote the postsingular set of $f$ by $P(f):=$ $\overline{\bigcup_{n>0} f^{n}(S(f))}$.

For more background on holomorphic dynamics we refer the reader to [14, 3].

2.2. Background on Riemann orbifolds. An orbifold is a space which is locally modeled on the quotient of an open set in $\mathbb{R}^{n}$ by the linear action of a finite group. For a general introduction see [26, § 13]. Throughout this article we will need only orbifolds modeled on Riemann surfaces, and for a more detailed introduction to this topic see e.g. [27, 10, 14].

Definition 2.1 (Riemann orbifold). A Riemann orbifold is a pair $(S, \nu)$, where $S$ is a Riemann surface and $\nu: S \rightarrow \mathbb{N}_{\geq 1}$ is a map called the ramification map, such that

$$
\{z \in S: \nu(z)>1\}
$$

is discrete. A point $z \in S$ with $\nu(z)>1$ is called a ramified or marked point. The signature of an orbifold is the list of values that the ramification map $\nu$ assumes at the ramified points, where a value is repeated as often as it occurs as $\nu(z)$ for some ramified point $z \in S$.

We will mainly require Riemann orbifolds whose underlying surface is a subset of $\widehat{\mathbb{C}}$. As usual, we will allow the surfaces to be disconnected; properties such as the type of the surface (hyperbolic, parabolic, elliptic) are understood componentwise.

A traditional Riemann surface can be regarded as a Riemann orbifold with ramification map $\nu \equiv 1$. Throughout this article, whenever we use the expression orbifold, we will always mean a Riemann orbifold.

Recall that for a holomorphic map $f: \widetilde{S} \rightarrow S$ between Riemann surfaces, the local degree $\operatorname{deg}\left(f, z_{0}\right)$ of $f$ at a point $z_{0} \in \widetilde{S}$ is the unique integer $n=n\left(z_{0}\right) \geq 1$, such that

$$
f(z)=f\left(z_{0}\right)+a_{n}\left(z-z_{0}\right)^{n}+(\text { higher terms })
$$

and $a_{n} \neq 0$. Thus $z_{0}$ is a critical or branch point of $f$ if and only if $n\left(z_{0}\right)>1$.

The map $f$ is called a branched covering map if every point in $S$ has a connected neighbourhood $U$ such that $f$ maps any component of $f^{-1}(U)$ onto $U$ as a proper map. Recall that a map $f: \widetilde{V} \rightarrow V$ is called proper if the preimage $f^{-1}(K)$ of any compact set $K \subset V$ is a compact subset of $\widetilde{V}$.

Definition 2.2 (Holomorphic map, covering). Let $\widetilde{\mathcal{O}}=(\widetilde{S}, \tilde{\nu})$ and $\mathcal{O}=(S, \nu)$ be Riemann orbifolds. A holomorphic map $f: \widetilde{\mathcal{O}} \rightarrow \mathcal{O}$ is a holomorphic map $f: \widetilde{S} \rightarrow S$ between the underlying Riemann surfaces such that, for each $z \in \widetilde{S}$,

$$
\nu(f(z)) \text { divides } \operatorname{deg}(f, z) \cdot \tilde{\nu}(z) .
$$

If $f: \widetilde{S} \rightarrow S$ is a branched covering map with $\nu(f(z))=\operatorname{deg}(f, z) \cdot \tilde{\nu}(z)$ for all $z \in \widetilde{S}$, then $f: \widetilde{\mathcal{O}} \rightarrow \mathcal{O}$ is an orbifold covering map. If additionally the surface $\widetilde{S}$ is simply-connected, then we call $\widetilde{\mathcal{O}}$ a universal covering orbifold of $\mathcal{O}$.

Remark. In the standard terminology, where an orbifold is defined via atlases and group actions, the definition of a holomorphic map $f$ between two orbifolds is equivalent to a "local lifting property"; if $f$ is a covering, then every such local lift can be chosen to be an embedding. For more details, see [10, A2]. 
Note that if $f: \widetilde{\mathcal{O}} \rightarrow \mathcal{O}$ is a covering, then this is not necessarily true for the map $f: \widetilde{S} \rightarrow S$ between the underlying surfaces. If $\widetilde{S} \subset S$, then the inclusion map $\widetilde{S} \hookrightarrow S$ is obviously holomorphic. In this setting, we can define the inclusion map $\widetilde{\mathcal{O}} \hookrightarrow \mathcal{O}$ on the corresponding orbifolds in the obvious manner. Note that this function does not have to be a holomorphic map in the sense of orbifolds.

Recall that by the Uniformization Theorem for Riemann surfaces, every Riemann surface has a universal cover that is conformally equivalent to either $\widehat{\mathbb{C}}, \mathbb{C}$ or $\mathbb{D}$. The following theorem tells us that the same is true for almost all Riemann orbifolds.

Theorem 2.3 (Uniformization of Riemann orbifolds, 10, Theorem A2]). Let $\mathcal{O}=$ $(S, \nu)$ be a Riemann orbifold. Then $\mathcal{O}$ has no universal covering orbifold if and only if $\mathcal{O}$ is isomorphic to $\widehat{\mathbb{C}}$ with signature $(l)$ or $(l, k)$, where $l \neq k$. In all other cases the universal cover is unique up to conformal isomorphism over the surface $S$ and hence given by either $\widehat{\mathbb{C}}, \mathbb{C}$ or $\mathbb{D}$.

In analogy to Riemann surfaces, we will call an orbifold $\mathcal{O}$ elliptic, parabolic or hyperbolic if it is covered by $\widehat{\mathbb{C}}, \mathbb{C}$ or $\mathbb{D}$, respectively.

Remark. Let $\widetilde{\mathcal{O}}, \mathcal{O}$ be orbifolds that have universal covers. Then a map $f: \widetilde{\mathcal{O}} \rightarrow \mathcal{O}$ is a covering if and only if it lifts to a conformal isomorphism between the universal covering spaces [14, Lemma E.2].

For a connected orbifold $\mathcal{O}=(S, \nu)$ the Euler characteristic $\chi(\mathcal{O})$ is given by the equation

$$
\chi(\mathcal{O}):=\chi(S)-\sum_{z \in S}\left(1-\frac{1}{\nu(z)}\right)
$$

where $\chi(S)$ denotes the Euler characteristic of the surface $S$. Note that ramified points cause a reduction of $\chi(\mathcal{O})$. As for Riemann surfaces, a Riemann orbifold with negative Euler characteristic is always hyperbolic. This also implies that, roughly speaking, most orbifolds are hyperbolic. For the precise list of spherical and parabolic orbifolds, see the details of [10, Theorem A2].

Let $C$ be the uniformized universal covering surface of $\mathcal{O}$ (i.e., $C \in\{\widehat{\mathbb{C}}, \mathbb{C}, \mathbb{D}\}$ ) and denote by $\rho_{C}(z)|d z|$ its unique complete conformal metric of constant curvature 1,0 or -1 , respectively. This metric is invariant under conformal automorphisms, hence it descends to a well-defined metric on the quotient $\mathcal{O}$. We call this metric the orbifold metric of $\mathcal{O}$ and denote it by $\rho_{\mathcal{O}}(w)|d w|$ (in terms of a local uniformizing parameter $w)$. Note that $\rho_{\mathcal{O}}(w)$ is nonzero and smooth except at the ramified points of $\mathcal{O}$, while at a ramified point, say $w_{0}$, the density has a singularity of the type $\left|w-w_{0}\right|^{(1-m) / m}$, where $m$ is the ramification value of $\mathcal{O}$ at $w_{0}$. More precisely, if we choose a local branched covering near 0, e.g., $z(w)=\left(w-w_{0}\right)^{m}$, then the induced metric $\rho(z(w))|d z / d w| \cdot|d w|$ is smooth and nonsingular throughout some neighbourhood of 0 in the $z$-plane.

Note that $\rho_{\mathcal{O}}(w)|d w|$ is again a complete metric with constant curvature 1,0 or -1 , respectively, everywhere except at the marked points (which are singularities of the curvature).

In this article we are mainly interested in hyperbolic Riemann orbifolds. The well-known Pick Theorem for hyperbolic surfaces generalizes to hyperbolic orbifolds as well and will be of great use for us. 
Theorem 2.4 ([27, Proposition 17.4]). A holomorphic map between two hyperbolic orbifolds can never increase distances as measured in the hyperbolic orbifold metric. Distances are strictly decreased, unless the map is a covering map; in this case it is a local isometry.

In particular, if $\widetilde{\mathcal{O}}$ and $\mathcal{O}$ are two orbifolds such that $\widetilde{\mathcal{O}} \hookrightarrow \mathcal{O}$ is holomorphic, then $\rho_{\widetilde{\mathcal{O}}}(z) \geq \rho_{\mathcal{O}}(z)$ for all $z \in \widetilde{\mathcal{O}}$.

2.3. Hyperbolic and subhyperbolic maps. Recall that our main result is the existence of a semiconjugacy between a strongly subhyperbolic and a disjoint type map on their Julia sets. We will now recall the definitions and present briefly the relevant dynamical properties of those types of functions.

Definition 2.5. A transcendental entire function $g$ is called hyperbolic if $P(g)$ is a compact subset of $\mathcal{F}(g)$.

It follows from classical results in holomorphic dynamics that the Fatou set of a hyperbolic (transcendental entire) map is nonempty and consists of attracting basins that correspond to finitely many attracting cycles.

The simple dynamical behaviour in attracting components allows us to control the forward orbit of any compact subset. We will frequently use the following statement. Since it is fairly basic and well known we will omit the proof; this can be found in [12, Proposition 3.1].

Proposition 2.6. Let $f$ be a transcendental entire map and denote by $A$ the set of all points whose orbit converges to an attracting periodic orbit of $f$. If $C \subset$ $A$ is compact, then there exist Jordan domains $J_{1}, \ldots, J_{n}$ compactly contained in pairwise different components of $A$, and an $\varepsilon>0$ such that

$$
f\left(\bigcup_{i=1}^{n} J_{i}\right) \cup D_{\varepsilon}\left(\overline{\bigcup_{k \geq 0} f^{k}(C)}\right) \Subset \bigcup_{i=1}^{n} J_{i} .
$$

A hyperbolic rational map is classically defined as a function which expands a conformal Riemannian metric defined on a neighbourhood of its Julia set. For entire transcendental maps one can give a similar description: it follows from Proposition 2.6 that if $g$ is hyperbolic, then there exists a bounded neighbourhood $D$ of the compact set $P(g)$ such that $\overline{g(D)} \subset D$. Note that by Montel's Theorem, $\left.g^{n}\right|_{D}$ is a normal family, hence $\mathbb{C} \backslash \bar{D}$ is a neighbourhood of $\mathcal{J}(g)$. Furthermore, the map $g: \mathbb{C} \backslash g^{-1}(\bar{D}) \rightarrow \mathbb{C} \backslash \bar{D}$ is then a covering map which uniformly expands the hyperbolic metric on the domain $\mathbb{C} \backslash \bar{D}$ [18, Lemma 5.1].

Note that not every hyperbolic rational map satisfies Definition 2.5 . By 14 , Theorem 19.1], a rational map is hyperbolic if and only if $P_{\mathcal{J}}=\emptyset$ or, equivalently, every critical point converges to an attracting periodic orbit. In certain cases (including all nonconstant polynomials), the point at $\infty$ can be such an attractor as well.

Definition 2.7. A hyperbolic transcendental entire map $g$ is said to be of disjoint type if $\mathcal{F}(g)$ is connected.

Since every component of the Fatou set of a hyperbolic transcendental entire map is simply-connected [7, Proposition 3, Theorem 1], it follows that the Fatou set of a disjoint type map is connected and simply-connected. 
Proposition 2.8. The following statements are equivalent:

(1) The map $g$ is of disjoint type.

(2) $g$ has a unique attracting fixed point and $P(g)$ is a compact subset of its immediate basin of attraction.

(3) There exists a Jordan domain $D \supset S(g)$ such that $\overline{g(D)} \subset D$.

Proof. Let $g$ be of disjoint type. In particular, $g$ is hyperbolic, and hence $P(g)$ is a compact subset of $\mathcal{F}(g)$. By definition, $\mathcal{F}(g)$ is connected, hence $P(g)$ is a compact subset of a completely invariant component of $\mathcal{F}(g)$, which can only be the immediate attracting basin of an attracting fixed point of $g$, showing that (1) implies (2).

We obtain statement (3) from (2) by setting $C:=S(g)$ in Proposition 2.6.

To see that (3) implies (1), let us choose a domain $D \supset S(g)$ such that $\overline{g(D)} \subset D$. By Montel's Theorem, $D$ is contained in a component of $\mathcal{F}(g)$, so, in particular, $g$ is hyperbolic and $\mathcal{F}(g)$ is the union of attracting basins. Since every immediate attracting basin contains at least one singular value [3, Theorem 7], $g$ has a unique attracting cycle which is a fixed point contained in $D$. Hence every point $z \in \mathcal{F}(g)$ is eventually mapped into $D$, showing that $\mathcal{F}(g)=\bigcup_{n \geq 0} g^{-n}(D)$. On the other hand, $g^{-(n+1)}(D) \supset g^{-n}(D) \supset S(g)$, hence every $g^{-(n+1)}(D)$ must be connected. So $\mathcal{F}(g)$ is the ascending union of connected sets and therefore connected.

Let $g$ be a transcendental entire map with bounded singular set $S(g)$, let $D \subset$ $\mathbb{C}$ be a bounded Jordan domain containing $S(g)$ and let $U:=\mathbb{C} \backslash \bar{D}$. Then $g$ : $g^{-1}(U) \rightarrow U$ is a covering map and each component $T$ of $g^{-1}(U)$ is a simplyconnected unbounded Jordan domain; we call every such domain $T$ a tract of $g$. Note that if $g$ is of disjoint type, then by Proposition 2.8 we can choose $D$ such that $g^{-1}(\mathbb{C} \backslash \bar{D})$ is disjoint from $\bar{D}$. Using such a domain one can easily prove the following property of the escaping set of a disjoint type map.

Proposition 2.9. Let $g$ be of disjoint type. Then $I(g)$ is disconnected.

Proof. Let $D \supset S(g)$ be a Jordan domain such that $g^{-1}(\mathbb{C} \backslash \bar{D})$ is disjoint from $\bar{D}$ and let $\alpha \subset g^{-1}(D) \backslash \bar{D}$ be a simple curve that connects $\partial D$ to $\infty$. (Indeed, such a curve always exists: every compact subset of the plane intersects only finitely many tracts, hence we can choose $\alpha$ to be the union of an arc to $\infty$ in $g^{-1}(\partial D)$ and a curve connecting this arc to $\partial D$ without intersecting the tracts.) The preimages of $\alpha$ split every tract of $g$ in simply-connected unbounded domains on which $g$ restricts as a conformal isomorphism; we call every such domain a fundamental domain. Since $\overline{g(D)} \cup g(\alpha) \subset D$, it follows that $I(g)$ is contained in the union of the fundamental domains.

By [6, Theorem 1], there exists a point $w \in I(g)$. Hence every fundamental domain must intersect $I(g)$ since it contains a preimage of $w$. Let $\widetilde{F}$ be an arbitrary but fixed fundamental domain and let $U$ denote the union of all fundamental domains other than $\widetilde{F}$. Then $U$ and $\widetilde{F}$ are two disjoint nonempty open sets whose union covers $I(g)$. Thus $I(g)$ is disconnected.

Definition 2.10. A transcendental entire function $f$ is called subhyperbolic if

(i) $P_{\mathcal{J}}:=P(f) \cap \mathcal{J}(f)$ is finite,

(ii) $P_{\mathcal{F}}:=P(f) \cap \mathcal{F}(f)$ is compact. 
It follows again from classical results that if $f$ is subhyperbolic, then $\mathcal{F}(f)$ is either empty or consists of attracting basins, and by condition (ii) there can be only finitely many attracting cycles.

By condition $(i)$, every singular value of $f$ in $\mathcal{J}(f)$ is preperiodic and every critical point in $\mathcal{J}(f)$ is strictly preperiodic. It also follows that $f$ has no indifferent periodic points in the Julia set. This means that each singular value in $\mathcal{J}(f)$ eventually lands on some repelling orbit. (For a proof of the above statements see e.g. [11, Proposition 2.5].)

A rational map is said to be subhyperbolic if it is expanding with respect to an orbifold metric. This characterization is equivalent to saying that every critical orbit is finite or converges to an attracting periodic orbit. Note that the postsingular set of a subhyperbolic rational function does not have to be bounded. However, for a general subhyperbolic transcendental map it is not possible to define an orbifold metric on a neighbourhood of the Julia which is expanded by the map. As we will see later, strongly subhyperbolic maps are exactly those maps for which we can construct the required orbifold metric. For completeness of this section, let us recall the definition of a strongly subhyperbolic map.

Definition 2.11. A subhyperbolic transcendental entire map $f$ is called strongly subhyperbolic if $\mathcal{J}(f) \cap A(f)=\emptyset$ and there is a constant $R<\infty$ such that $\operatorname{deg}(f, z)<R$ holds for all $z \in \mathcal{J}(f)$.

\section{Subhyperbolic maps AND DYNAmicAlly ASsociated ORBIFOLDS}

Let $f$ be a strongly subhyperbolic map. The first step towards the proof of Theorem 1.2 is to find hyperbolic orbifolds

$$
\mathcal{O}_{f}=\left(S_{f}, \nu_{f}\right) \quad \text { and } \quad \widetilde{\mathcal{O}}_{f}=\left(\widetilde{S}_{f}, \tilde{\nu}_{f}\right)
$$

with simple geometries such that $f: \widetilde{\mathcal{O}}_{f} \rightarrow \mathcal{O}_{f}$ is expanding with respect to the hyperbolic metric on $\mathcal{O}_{f}$. We will make use of the following simple observation.

Proposition 3.1. Let $\mathcal{O}=(S, \nu)$ and $\widetilde{\mathcal{O}}=(\widetilde{S}, \tilde{\nu})$ be hyperbolic orbifolds with metrics $\rho_{\mathcal{O}}(z)|d z|$ and $\rho_{\widetilde{\mathcal{O}}}(z)|d z|$, respectively. Let $f: \widetilde{\mathcal{O}} \rightarrow \mathcal{O}$ be a covering map and assume that the inclusion $\widetilde{\mathcal{O}} \hookrightarrow \mathcal{O}$ is holomorphic but not a covering. Then

$$
\|D f(z)\|_{\mathcal{O}}:=\left|f^{\prime}(z)\right| \cdot \frac{\rho_{\mathcal{O}}(f(z))}{\rho_{\mathcal{O}}(z)}>1
$$

wherever this is defined.

Proof. By Theorem $2.4 f$ is a local isometry, hence

$$
\rho_{\widetilde{\mathcal{O}}}(z)=\rho_{\mathcal{O}}(f(z)) \cdot\left|f^{\prime}(z)\right| .
$$

Since the inclusion is only holomorphic, it is a strict contraction, and hence $\rho_{\widetilde{\mathcal{O}}}(z)>$ $\rho_{\mathcal{O}}(z)$. So altogether,

$$
\rho_{\widetilde{\mathcal{O}}}(z)=\rho_{\mathcal{O}}(f(z)) \cdot\left|f^{\prime}(z)\right|>\rho_{\mathcal{O}}(z)
$$

implying that $\|D f(z)\|_{\mathcal{O}}>1$. 
3.1. Construction of $\mathcal{O}_{f}$ and $\widetilde{\mathcal{O}}_{f}$ for a strongly subhyperbolic map $f$. Our first goal is to find hyperbolic orbifolds $\mathcal{O}_{f}$ and $\widetilde{\mathcal{O}}_{f}$ such that the assumptions of Proposition 3.1 are satisfied. For the maps we consider there is a natural way to construct such orbifolds, and we will follow the approach of Douady and Hubbard for subhyperbolic rational maps [4. However, it is possible that the orbifolds obtained by this canonical construction have geometries which do not allow us to compute sufficiently good estimates of the corresponding metrics. To solve this problem, we will modify the obtained orbifolds in a second step.

Proposition 3.2 (Canonical orbifolds). Let $f$ be a strongly subhyperbolic function. Then there exist orbifolds $\mathcal{O}_{f}^{*}=\left(S_{f}^{*}, \nu_{f}^{*}\right)$ and $\widetilde{\mathcal{O}}_{f}^{*}=\left(\widetilde{S}_{f}^{*}, \tilde{\nu}_{f}^{*}\right)$ with the following properties:

(a) $S_{f}^{*}=\mathbb{C} \backslash K$, where $K=\emptyset$ or $K$ is the finite union of closed Jordan domains, and $\widetilde{S}_{f}^{*}$ and $S_{f}^{*}$ have no common boundary points.

(b) The set $B_{f}^{*}$ of ramified points of $\mathcal{O}_{f}^{*}$ is finite and contains $P_{\mathcal{J}}$.

(c) $\mathcal{J}(f) \subset S_{f}^{*}$ while $P_{\mathcal{F}} \cap S_{f}^{*}=\emptyset$.

(d) $\mathcal{O}_{f}^{*}$ is hyperbolic.

(e) $f: \widetilde{\mathcal{O}}_{f}^{*} \rightarrow \mathcal{O}_{f}^{*}$ is a covering map.

$(f)$ The inclusion $\widetilde{\mathcal{O}}_{f}^{*} \hookrightarrow \mathcal{O}_{f}^{*}$ is holomorphic but not a covering map.

We will say that two orbifolds are canonical for $f$ if they satisfy $(a)-(f)$.

Note that statement $(f)$ in particular implies that $\widetilde{S}_{f}^{*} \subset S_{f}^{*}$.

Proof. We start with the construction of $S_{f}^{*}$. If $\mathcal{F}(f)=\emptyset$, then define $K:=\emptyset$ and $S_{f}^{*}:=\mathbb{C}$. Otherwise, the Fatou set of $f$ consists of attracting basins only, and by Proposition 2.6 we can find Jordan domains $J_{1}, \ldots, J_{n}$ such that $J:=\bigcup_{i=1}^{n} J_{i}$ satisfies $D_{\varepsilon}\left(P_{\mathcal{F}}\right) \cup f(J) \Subset J \Subset \mathcal{F}(f)$ for some $\varepsilon>0$. We choose a set $J$ with this property and define

$$
K:=\bar{J} \quad \text { and } \quad S_{f}^{*}:=\mathbb{C} \backslash K .
$$

Note that $S_{f}^{*}$ is connected and that $\mathcal{J}(f)$ is entirely contained in $S_{f}^{*}$.

We define the ramification map to be

$$
\nu_{f}^{*}: S_{f}^{*} \rightarrow \mathbb{N}_{\geq 1}, z \mapsto \nu_{f}^{*}(z):=\operatorname{lcm}\left\{\operatorname{deg}\left(f^{m}, w\right), \text { where } f^{m}(w)=z\right\} .
$$

Let $\mathcal{O}_{f}^{*}=\left(S_{f}^{*}, \nu_{f}^{*}\right)$. By construction, $\nu_{f}^{*}(z)>1$ if and only if $z \in P_{\mathcal{J}}$, implying that $B_{f}^{*}$ is finite. Furthermore, no critical point $c \in S_{f}^{*}$ belongs to a periodic cycle, and since we have assumed that the local degree at all points in $\mathcal{J}(f)$ is uniformly bounded, the ramification value $\nu_{f}^{*}(z)$ is necessarily a finite number for each $z \in S_{f}^{*}$. Hence $\mathcal{O}_{f}^{*}=\left(S_{f}^{*}, \nu_{f}^{*}\right)$ is a Riemann orbifold. Note that $\mathcal{O}_{f}^{*}$ satisfies statements $(b)$ and $(c)$.

Define

$$
\widetilde{S}_{f}^{*}:=f^{-1}\left(S_{f}^{*}\right) \quad \text { and } \quad \tilde{\nu}_{f}^{*}: \widetilde{S}_{f}^{*} \rightarrow \mathbb{N}_{\geq 1}, z \mapsto \tilde{\nu}_{f}^{*}(z):=\frac{\nu_{f}^{*}(f(z))}{\operatorname{deg}(f, z)}
$$

By equation (3.2), $\tilde{\nu}_{f}^{*}(z)$ is a positive integer for every $z \in \widetilde{S}_{f}^{*}$, and by the Identity Theorem the set of points $z \in \widetilde{S}_{f}^{*}$ with $\tilde{\nu}_{f}^{*}(z)>1$ is discrete. Hence $\widetilde{\mathcal{O}}_{f}^{*}=\left(\widetilde{S}_{f}^{*}, \tilde{\nu}_{f}^{*}\right)$ is a Riemann orbifold. Furthermore, if $S_{f}^{*}=\mathbb{C}$, then $\widetilde{S}_{f}^{*}=\mathbb{C}$ as well, and otherwise 
the properties of the set $K$ imply that the closure of $\widetilde{S}_{f}^{*}$ is contained in $S_{f}^{*}$. So we have proved statement $(a)$.

Next we will prove that $\mathcal{O}_{f}^{*}$ is hyperbolic. If $S_{f}^{*} \neq \mathbb{C}$, then by construction $S_{f}^{*}$ is a hyperbolic domain. Since $\chi\left(\mathcal{O}_{f}^{*}\right) \leq \chi\left(S_{f}^{*}\right)$, the orbifold $\mathcal{O}_{f}^{*}$ must then be hyperbolic as well. Now let $S_{f}^{*}=\mathbb{C}$. By $\left[10\right.$, Theorem A2], $\mathcal{O}_{f}^{*}$ is either hyperbolic or parabolic, and in the latter case its signature is either $(n)$ or $(2,2)$.

Assume first that $\mathcal{O}_{f}^{*}$ has signature $(n)$. Then $f$ has only one singular value, say at $z=0$. It follows from a covering space argument that $f(z)=\exp (a z+b)$, where $a \in \mathbb{C}^{*}$ and $b \in \mathbb{C}$. But then 0 is an asymptotic value (and since it is omitted, it cannot be a fixed point either), yielding a contradiction.

Let us now assume that $\mathcal{O}_{f}^{*}$ has signature $(2,2)$. By the previous argument, $f$ has exactly two singular values $v_{1}$ and $v_{2}$ which are necessarily critical values, and any of their preimages is either a critical point of local degree two or a regular point. Signature $(2,2)$ also implies that $P(f)=S(f)$, meaning that both critical values are either fixed points of $f$ or they form a two-cycle. Since $\mathcal{F}(f)=\emptyset, v_{1}$ and $v_{2}$ are both repelling. In particular, $\operatorname{deg}\left(f, v_{1}\right)=\operatorname{deg}\left(f, v_{2}\right)=1$. Let $\mathcal{O}_{f}^{\prime}=\left(S_{f}^{\prime}, \nu_{f}^{\prime}\right)$ be the orbifold which has exactly the regular preimages of $v_{1}$ and $v_{2}$ as ramified points, assigning them the ramification value two. Clearly, $\nu_{f}^{\prime}\left(v_{1}\right)=\nu_{f}^{\prime}\left(v_{2}\right)=2$. Then $f: \mathcal{O}_{f}^{\prime} \rightarrow \mathcal{O}_{f}^{*}$ is a covering map, and since $\mathcal{O}_{f}^{*}$ is parabolic, so is $\mathcal{O}_{f}^{\prime}$, which means that $v_{1}$ and $v_{2}$ are the only ramified points in $\mathcal{O}_{f}^{\prime}$. Hence $\mathcal{O}_{f}^{\prime}=\mathcal{O}_{f}^{*}$. By conformal conjugacy we can assume that $v_{1}=1$ and $v_{2}=-1$. Then the map $\mathbb{C} \rightarrow \mathcal{O}_{f}^{*}, z \mapsto \cos (z)$ is a universal covering map. Since $f: \mathcal{O}_{f}^{*} \rightarrow \mathcal{O}_{f}^{*}$ is a covering map, it lifts to a conformal $\mathbb{C}$-isomorphism $g(z)=a z+b, a \neq 0$, yielding the relation

$$
f(\cos (z))=\cos (a z+b) .
$$

By periodicity and symmetry of the cosine map, $a \in \mathbb{Z} \backslash\{0\}$ and $b \in \pi \mathbb{Z}$. But this means that $f$ or $-f$ is a Chebyshev polynomial, contradicting the fact that $f$ is transcendental. Hence $\mathcal{O}_{f}^{*}$ is hyperbolic, and we have proved $(d)$.

Since $A(f) \cap S_{f}^{*}=\emptyset$, the map $f: \widetilde{S}_{f}^{*} \rightarrow S_{f}^{*}$ is a branched covering. Furthermore,

$$
\operatorname{deg}(f, z) \cdot \tilde{\nu}_{f}^{*}(z)=\operatorname{deg}(f, z) \cdot \frac{\nu_{f}^{*}(f(z))}{\operatorname{deg}(f, z)}=\nu_{f}^{*}(f(z)),
$$

hence $f: \widetilde{\mathcal{O}}_{f}^{*} \rightarrow \mathcal{O}_{f}^{*}$ is an orbifold covering map, proving statement $(e)$.

We will now show that the inclusion $\widetilde{\mathcal{O}}_{f}^{*} \hookrightarrow \mathcal{O}_{f}^{*}$ is holomorphic but not a covering. Let $z \in S_{f}^{*}$. Observe that the definition of $\nu_{f}^{*}$ (see equation (3.2)) together with the fact that for any point $\omega \in \mathbb{C}$ the local degree of an iterate $f^{m}$ of $f$ is given by $\operatorname{deg}\left(f^{m}, \omega\right)=\operatorname{deg}(f, \omega) \cdot \operatorname{deg}(f, f(\omega)) \cdot \ldots \cdot \operatorname{deg}\left(f, f^{m-1}(\omega)\right)$ implies that $\nu_{f}^{*}(z)$. $\operatorname{deg}(f, z)$ divides $\nu_{f}^{*}(f(z))$. Since $f: \widetilde{\mathcal{O}}_{f}^{*} \rightarrow \mathcal{O}_{f}^{*}$ is a covering, $\tilde{\nu}_{f}^{*}(z)=\nu_{f}^{*}(z)$. $\operatorname{deg}(f, z)=\nu_{f}^{*}(f(z))$. Hence $\nu_{f}^{*}(z)$ divides $\tilde{\nu}_{f}^{*}(z)$ and this proves that the inclusion $\widetilde{\mathcal{O}}_{f}^{*} \hookrightarrow \mathcal{O}_{f}^{*}$ is a holomorphic map.

We will now show that the inclusion $\widetilde{\mathcal{O}}_{f}^{*} \hookrightarrow \mathcal{O}_{f}^{*}$ is not an orbifold covering map. This is obvious if $S_{f}^{*} \neq \mathbb{C}$, since $\widetilde{S}_{f}^{*} \subsetneq S_{f}^{*}$, so let us assume that $S_{f}^{*}=\mathbb{C}$. It follows from the discussion on hyperbolicity of $\mathcal{O}_{f}^{*}$ that the signature of $\mathcal{O}_{f}^{*}$ has either at least three entries or it is of the form $(m, n)$ with $n \geq 3$. We will show that $\widetilde{\mathcal{O}}_{f}^{*}$ contains infinitely many points with $\tilde{\nu}_{f}^{*}(z)>1$; this will imply our claim since the 
set of ramified points of $\mathcal{O}_{f}^{*}$ is finite. To do this, let us consider the function

$$
\theta(a, f):=1-\limsup _{r \rightarrow \infty} \frac{\overline{\mathrm{N}}(r, a, f)}{\mathrm{T}(r, f)},
$$

where $T(r, f)$ denotes the Nevanlinna characteristic of $f$ and $\overline{\mathrm{N}}(r, a, f)$ the counting function of $a$-points of $f$ without multiplicity (for more information see [15, X.§3]). If $a$ has only finitely many simple preimages under $f$, then $\theta(a, f) \geq \frac{1}{2}$ [15, X. §3.238]. On the other hand, $\sum_{a \in \mathbb{C}} \theta(a, f) \leq 1$ whenever $f$ is transcendental entire [15. X. §3.237]. Hence for a given function, there can be at most two points with only finitely many simple preimages. So if the signature of $\mathcal{O}_{f}^{*}$ has more than two entries, then equation (3.3) implies that $\widetilde{\mathcal{O}}_{f}^{*}$ has infinitely many ramified points.

Let us now consider the case when the signature of $\mathcal{O}_{f}^{*}$ is of the form $(m, n)$ with $n \geq 3$, and denote by $a_{1}$ the point with ramification $m$ and by $a_{2}$ the point with ramification $n$. If both $a_{1}$ and $a_{2}$ have only finitely many simple preimages under $f$, then by the previous arguments, we have $\theta\left(a_{1}\right)=\theta\left(a_{2}\right)=1 / 2$. By the definition of $\theta$, for every $i=1,2$ there must be infinitely many points at which the degree of $f$ is at most 2. Let $z_{k}$ be such a sequence of points corresponding to $a_{2}$. Since $\nu_{f}^{*}\left(a_{2}\right)=n \geq 3$, it follows again by equation (3.3) that $\tilde{\nu}_{f}^{*}\left(z_{k}\right)>1$, yielding an infinite set of ramified points in $\widetilde{\mathcal{O}}_{f}^{*}$.

Remarks. Let $\widetilde{\mathcal{O}}_{f}^{*}$ and $\mathcal{O}_{f}^{*}$ be canonical for $f$. Note that $\widetilde{\mathcal{O}}_{f}^{*}$ is usually not connected. However, if $f$ has finitely many tracts over $\infty$, e.g., if $f$ has finite order, then the number of components of $\widetilde{\mathcal{O}}_{f}^{*}$ is finite. Observe also that it follows from Proposition 3.2 $(f)$ that the set $B_{f}^{*}$ of ramified points of $\mathcal{O}_{f}^{*}$ satisfies $f\left(B_{f}^{*}\right) \subset B_{f}^{*}$. Also note that a punctured neighbourhood of $\infty$ is holomorphically embedded in $\mathcal{O}_{f}^{*}$.

We have proved that one can achieve a stronger statement than $(b)$; in fact, our construction yields an orbifold $\mathcal{O}_{f}^{*}$ for which the set of ramified points equals $P_{\mathcal{J}}$ (see equation (3.2)). However, we have stated the weaker requirement since the orbifolds which we will obtain after modification of canonical orbifolds will in general have a slightly larger set of ramified points.

Orbifolds $\widetilde{\mathcal{O}}_{f}^{*}$ and $\mathcal{O}_{f}^{*}$, constructed as in the previous proof, are minimal in the following sense: any pair of orbifolds $(\widetilde{\mathcal{U}}, \mathcal{U})$ such that $\mathcal{U}$ contains $\mathcal{J}(f)$ while $f$ : $\widetilde{\mathcal{U}} \rightarrow \mathcal{U}$ is a covering map and $\widetilde{\mathcal{U}} \hookrightarrow \mathcal{U}$ is holomorphic forces ramification of all points in $P_{\mathcal{J}}$. The definition of the ramification function as in equation (3.2) using least common multiples yields the smallest possible ramification numbers.

In the last part of the proof of Proposition 3.2 we have shown the following: if $S_{f}^{*}$ is hyperbolic, then $S_{f}^{*} \backslash \widetilde{S}_{f}^{*}$ is unbounded, but even if $\widetilde{S}_{f}^{*}=S_{f}^{*}=\mathbb{C}$, then $\widetilde{\mathcal{O}}_{f}^{*}$ contains an infinite sequence of ramified points, contrary to $\mathcal{O}_{f}^{*}$. However, we do not gain much information on the geometry of these points. In order to obtain sufficiently strong geometric estimates for the metric of $\widetilde{\mathcal{O}}_{f}^{*}$ we will modify canonical orbifolds in the following way.

Definition 3.3. A pair of orbifolds $\left(\widetilde{\mathcal{O}}_{f}, \mathcal{O}_{f}\right)$ will be called dynamically associated to $f$ if the following properties hold:

(i) $\widetilde{\mathcal{O}}_{f}$ and $\mathcal{O}_{f}$ are canonical,

(ii) there exists a point $p \in S_{f} \backslash S(f)$ such that $\nu_{f}(p)=2 k$ for some $k>1$. 
Proposition 3.4. Let $f$ be strongly subhyperbolic. Then there exists a pair of orbifolds $\left(\widetilde{\mathcal{O}}_{f}, \mathcal{O}_{f}\right)$ dynamically associated to $f$.

Proof. Let $\widetilde{\mathcal{O}}_{f}^{*}$ and $\mathcal{O}_{f}^{*}$ be canonical for $f$, constructed as in the proof of Proposition 3.2. If there is a point $p \in P_{\mathcal{J}} \backslash S(f)$ such that for every point $z \in \operatorname{Crit}(f)$ with $f^{n}(z)=p$ there exists $l \geq 1$ with $\operatorname{deg}\left(f^{n}, z\right)=2 l$, then it follows from equation (3.2) that $\left(\widetilde{\mathcal{O}}_{f}^{*}, \mathcal{O}_{f}^{*}\right)$ is dynamically associated to $f$. If there is no such point, then we pick a repelling fixed point $p$ of $f$ and define $\mathcal{O}_{f}:=\left(S_{f}^{*}, \nu_{f}\right)$, where

$$
\nu_{f}(w):= \begin{cases}\nu_{f}^{*}(w) & \text { if } w \neq p \\ 2 & \text { if } w=p .\end{cases}
$$

Note that such a point can always be found since maps with bounded sets of singular values have infinitely many fixed points [9, Theorem 2]. For the definition of $\widetilde{\mathcal{O}}_{f}$ we use the recipe from equation (3.3), meaning we define $\widetilde{\mathcal{O}}_{f}:=\left(\widetilde{S}_{f}^{*}, \tilde{\nu}_{f}\right)$, where

$$
\tilde{\nu}_{f}(z):= \begin{cases}\tilde{\nu}_{f}^{*}(z) & \text { if } f(z) \neq p \\ 2 & \text { if } f(z)=p .\end{cases}
$$

It follows immediately that $\left(\widetilde{\mathcal{O}}_{f}, \mathcal{O}_{f}\right)$ is dynamically associated to $f$.

Corollary 3.5. Let $f$ be strongly subhyperbolic and $\left(\widetilde{\mathcal{O}}_{f}, \mathcal{O}_{f}\right)$ dynamically associated to $f$. Then there is a constant $K>1$ and a sequence of points $z_{i} \rightarrow \infty$ for which $\tilde{\nu}_{f}\left(z_{i}\right)$ is a multiple of 2 , such that $\left|z_{i}\right|<\left|z_{i+1}\right| \leq K\left|z_{i}\right|$ holds for all $i$.

Proof. Let $p \in S_{f} \backslash S(f)$ be a point such that $\nu_{f}(p)$ is a multiple of 2 and let $\gamma$ be a Jordan curve in $\mathbb{C}$ such that the bounded component of $\mathbb{C} \backslash \gamma$ contains $S(f)$ but not $p$. The components of the preimage of the unbounded component of $\mathbb{C} \backslash \gamma$ are tracts of $f$ and every such tract contains infinitely many preimages of $p$. Let $z_{i}$ denote the preimages of $p$ lying in one such (arbitrary but fixed) tract. Using standard estimates on the hyperbolic metric in a simply-connected domain 14. Corollary A.8], one easily deduces that there is a constant $K>1$ such that $\left|z_{i}\right|<\left|z_{i+1}\right| \leq K\left|z_{i}\right|$ holds for infinitely many $i$. (For details, see e.g. [18, Proof of Lemma 5.1] or [11, Proof of Proposition 3.4].) However, since all but finitely many $z_{i}$ are regular points of $f$, it follows that $\tilde{\nu}_{f}\left(z_{i}\right)=\nu_{f}(p)$ and this is by assumption a multiple of 2 .

Remark. In order to obtain a sequence of ramified points in $\widetilde{\mathcal{O}}_{f}$ as in Corollary 3.5 we could also have ramified a repelling periodic orbit in $\mathcal{O}_{f}$ of period larger than 1. It will also become clear that for our purposes a sequence of ramified values in $\widetilde{\mathcal{O}}_{f}$ with higher ramification would be sufficient as well, but we choose ramification 2 since it is optimal for estimates in the spirit of Theorem 1.5 .

Notation. For a pair $\left(\widetilde{\mathcal{O}}_{f}, \mathcal{O}_{f}\right)$ of orbifolds dynamically associated to $f$, we denote by $\tilde{\rho}_{f}$ and $\rho_{f}$ the densities of the hyperbolic metrics of $\widetilde{\mathcal{O}}_{f}$ and $\mathcal{O}_{f}$, respectively.

We conclude this section with the following simple observation which justifies our restriction to strongly subhyperbolic maps.

Proposition 3.6. Let $f$ be a subhyperbolic map for which there is a pair of canonical orbifolds. Then $f$ is strongly subhyperbolic. 
Proof. If $w$ is an asymptotic value of $f$ and $D$ is any neighbourhood of $w$, then the restriction $f: f^{-1}(D) \rightarrow D$ is not a proper map. Hence if $\mathcal{J}(f) \cap A(f) \neq \emptyset$, then there is no domain $U \supset \mathcal{J}(f)$ such that $f: f^{-1}(U) \rightarrow U$ is a (branched) covering map.

Now assume that $f$ has a critical value $w \in \mathcal{J}(f)$ such that for every $n \in \mathbb{N}$ there exists a point $z_{n}$ with $f\left(z_{n}\right)=w$ and $\operatorname{deg}\left(f, z_{n}\right) \geq n$. If there was a pair $\left(\widetilde{\mathcal{O}}_{f}^{*}, \mathcal{O}_{f}^{*}\right)$ of canonical orbifolds, then Proposition $3.2(e)$ would imply that $w$ is a puncture of $\mathcal{O}_{f}^{*}$, contradicting the fact that $\mathcal{J}(f) \subset S_{f}^{*}$.

\section{UNIFORM EXPANSION}

Let $f$ be a strongly subhyperbolic map and let $\left(\widetilde{\mathcal{O}}_{f}, \mathcal{O}_{f}\right)$ be dynamically associated to $f$. By Proposition 3.1

$$
\|D f(z)\|_{\mathcal{O}_{f}}=\left|f^{\prime}(z)\right| \cdot \frac{\rho_{f}(f(z))}{\rho_{f}(z)}>1
$$

wherever defined, so in particular for all $z \in \widetilde{\mathcal{O}}_{f}$.

Our goal is to show that the expansion of $f$ is uniform.

Theorem 4.1 (Uniform expansion). Let $f$ be a strongly subhyperbolic map and let $\left(\widetilde{\mathcal{O}}_{f}, \mathcal{O}_{f}\right)$ be dynamically associated to $f$. Then there is a constant $E>1$ such that

$$
\|D f(z)\|_{\mathcal{O}_{f}} \geq E
$$

for all $z \in \widetilde{\mathcal{O}}_{f}$.

The remainder of Section 4 is devoted to the proof of Theorem 4.1 ,

4.1. Continuity of orbifold metrics. We want to show the following continuity statement: Let $\mathcal{O}$ be a Riemann orbifold, let $p$ be a regular and $q$ a ramified point of $\mathcal{O}$. Then the value of the density map $\rho_{\mathcal{O}}$ of the orbifold metric at $p$ depends continuously on $q$, i.e., if we perturb the point $q$ slightly, then the density of the corresponding orbifold metric at the point $p$ will also undergo only a small change.

This statement is surely not new, but we were not able to locate a reference. Hence we include a proof for completeness. We will restrict ourselves to orbifolds whose underlying surface is a subset of the sphere. The proof in the general case, where the underlying surface of $\mathcal{O}$ is an arbitrary Riemann surface, can be derived using exactly the same arguments, with the additional step of taking charts.

So let $S \subset \widehat{\mathbb{C}}$ and let $\mathcal{O}=(S, \nu)$. Denote by $B$ the set of ramified points of $\mathcal{O}$. Let $n>1$ be an arbitrary but fixed integer. For a point $q \in S \backslash B$ we define a new orbifold $\mathcal{O}_{q}=\left(S, \nu_{q}\right)$, where

$$
\nu_{q}(z)= \begin{cases}\nu(z) & \text { if } z \neq q \\ n & \text { if } z=q .\end{cases}
$$

Furthermore, we assume that every such orbifold has a universal cover (hence we exclude the case when $S$ is a sphere and the set $B$ is either empty or consists of only one point with ramification value $m \neq n$ ). Note that any two such orbifolds $\mathcal{O}_{q}$ and $\mathcal{O}_{\tilde{q}}$ have the same signature and hence the same uniformized universal cover. Let us denote the density of the orbifold metric on $\mathcal{O}_{q}$ by $\rho_{q}$.

For a point $p \in S \backslash B$ we define the map

$$
M_{p}: S \backslash(B \cup\{p\}) \rightarrow(0, \infty], \quad q \mapsto \rho_{q}(p) .
$$


Theorem 4.2 (Continuity of orbifold metrics). Let $p \in S \backslash B$ be arbitrary but fixed. Then the map $M_{p}$ is continuous at every point in $S \backslash(B \cup\{p\})$.

Proof. Let $q^{*} \in S \backslash(B \cup\{p\})$ be an arbitrary but fixed point. We want to show that $M_{p}$ is continuous at $q^{*}$.

Pick a sufficiently small Jordan domain $D \ni q^{*}$ such that $\bar{D} \cap(B \cup\{p\})=\emptyset$. Let $a$ be a point in $S \backslash(\bar{D} \cup B \cup\{p\})$. By conformal conjugacy, we can assume that $a=0$.

For two points $z_{1}, z_{2} \in D$ let us denote by $d_{D}\left(z_{1}, z_{2}\right)$ the distance between $z_{1}$ and $z_{2}$ measured in the hyperbolic metric of $D$. For a point $q \in D$ consider the unique Riemann map $H_{q}: D \rightarrow \mathbb{H}$ which maps $q^{*} \mapsto i$ and $q \mapsto h_{q} i$, where $h_{q}:=\mathrm{e}^{d_{D}\left(q^{*}, q\right)}$. Let $L_{q}: \mathbb{H} \rightarrow \mathbb{H},(x+i y) \mapsto x+h_{q} y i$. Then $L_{q}$ is a $h_{q}$-quasiconformal self-map of HI. Define

$$
\varphi_{q}: D \rightarrow D, \quad z \mapsto H_{q} \circ L_{q} \circ H_{q}^{-1}(z)
$$

It is easy to see that $\varphi_{q}$ extends continuously to the complement of $D$ as the identity map and that the extended map, which we will also denote by $\varphi_{q}$, is $h_{q^{-}}$ quasiconformal (see e.g. [8, Lemma 5.2.3]). Observe that $\varphi_{q} \rightarrow \varphi_{q^{*}} \equiv$ id as $q \rightarrow q^{*}$.

Let $C$ be the uniformized universal covering surface of $\mathcal{O}_{q^{*}}$ and $\mathcal{O}_{q}$ and let $\pi_{q^{*}}$ : $C \rightarrow \mathcal{O}_{q^{*}}$ and $\pi_{q}: C \rightarrow \mathcal{O}_{q}$ be universal covering maps, both normalized such that $\pi_{q^{*}}(0)=\pi_{q}(0)=0$ and $\pi_{q^{*}}^{\prime}(0)=\pi_{q}^{\prime}(0)$. Considered as a map between orbifolds, $\varphi_{q}: \mathcal{O}_{q^{*}} \rightarrow \mathcal{O}_{q}$ is a homeomorphism and hence can be lifted to a homeomorphism on $C$. From now on we will assume that $C=\mathbb{D}$ since the other two cases follow by the same strategy, using even simpler calculations.

Claim. There is a unique lift $\tilde{\varphi}_{q}: \mathbb{D} \rightarrow \mathbb{D}$ of $\varphi_{q}$ such that $\tilde{\varphi}_{q}(0)=0$.

Proof of the Claim. Let $G_{q}$ denote the covering group of $\mathbb{D}$ over $\mathcal{O}_{q}$ and let $\tilde{\varphi}_{q}, \tilde{\tilde{\varphi}}_{q}$ be lifts of $\varphi_{q}$ that fix 0 . Then there exists a mapping $h \in G_{q}$ such that $\tilde{\tilde{\varphi}}_{q}(z)=$ $h\left(\tilde{\varphi}_{q}(z)\right)$ holds for all $z \in \mathbb{D}$. It follows from our assumption that $h(0)=0$, hence $h \in \operatorname{Stab}(0) \subset G_{q}$, where $\operatorname{Stab}(0)$ denotes the stabilizer of 0 in $G_{q}$. But $\pi_{q}(0)=0$ and 0 is a nonramified point of $\mathcal{O}_{q}$, which means that $\operatorname{Stab}(0) \subset G_{q}$ is trivial. Hence $h \equiv \mathrm{id}$ and $\tilde{\varphi}_{q} \equiv \tilde{\tilde{\varphi}}_{q}$.

We have the following commutative diagram:

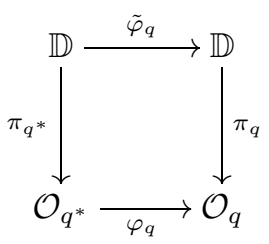

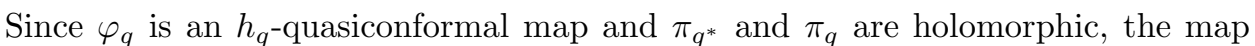
$\tilde{\varphi}_{q}: \mathbb{D} \rightarrow \mathbb{D}$ is also $h_{q}$-quasiconformal. Moreover, $\tilde{\varphi}_{q}$ is conformal when restricted to the set $\Omega:=\mathbb{D} \backslash \pi_{q^{*}}^{-1}(D)$, so in particular in a sufficiently small neighbourhood of any point in the set $\left\{\pi_{q^{*}}^{-1}(p)\right\}$.

Furthermore, the lifts $\tilde{\varphi}_{q}$ converge to $\tilde{\varphi}_{q^{*}}$ (locally uniformly) as $q \rightarrow q^{*}$ and, due to the chosen normalization, $\tilde{\varphi}_{q^{*}} \equiv$ id $\left.\right|_{\mathbb{D}}$. Moreover, when restricted to $\Omega$, the maps converge in the $C^{1}$-norm, hence $\left.\left.\left(\tilde{\varphi}_{q}\right)^{\prime}\right|_{\Omega} \rightarrow\left(\tilde{\varphi}_{q^{*}}\right)^{\prime}\right|_{\Omega}$ when $q \rightarrow q^{*}$.

By the previous diagram we can write $\pi_{q}(z)=\left(\varphi_{q} \circ \pi_{q^{*}} \circ \tilde{\varphi}_{q}^{-1}\right)(z)$ for every $z \in \mathbb{D}$. Recall that if $w \in S$ and $z_{q} \in\left\{\pi_{q}^{-1}(w)\right\}$, then the value of the density function $\rho_{q}$ at 
$w$ is given by $\rho_{q}(w)=\rho_{\mathbb{D}}\left(z_{q}\right) \cdot\left|\pi_{q}^{\prime}\left(z_{q}\right)\right|^{-1}$, and this does not depend on the choice of the preimage of $w$. Similarly, if $z_{q^{*}} \in\left\{\pi_{q^{*}}^{-1}(w)\right\}$, then $\rho_{q^{*}}(w)=\rho_{\mathbb{D}}\left(z_{q^{*}}\right) \cdot\left|\pi_{q^{*}}^{\prime}\left(z_{q^{*}}\right)\right|^{-1}$. Hence,

$$
\left|\rho_{q^{*}}(w)-\rho_{q}(w)\right|=\left|\frac{\rho_{\mathbb{D}}\left(z_{q^{*}}\right)}{\left|\pi_{q^{*}}^{\prime}\left(z_{q^{*}}\right)\right|}-\frac{\rho_{\mathbb{D}}\left(z_{q}\right)}{\left|\pi_{q}^{\prime}\left(z_{q}\right)\right|}\right| .
$$

First observe that $\varphi_{q}(p)=p$ since $p \in S \backslash D$. Let us fix a point $p_{q} \in\left\{\pi_{q}^{-1}(p)\right\}$. Then

$$
p=\pi_{q}\left(p_{q}\right)=\left(\varphi_{q} \circ \pi_{q^{*}} \circ \tilde{\varphi}_{q}^{-1}\right)\left(p_{q}\right)=\left(\pi_{q^{*}} \circ \tilde{\varphi}_{q}^{-1}\right)\left(p_{q}\right) .
$$

Let $p_{q^{*}} \in\left\{\pi_{q^{*}}^{-1}(p)\right\}$ be the unique point such that $p_{q}=\tilde{\varphi}_{q}\left(p_{q^{*}}\right)$. We obtain

$$
\begin{aligned}
& \pi_{q}^{\prime}\left(p_{q}\right)=\varphi_{q}^{\prime}\left(\left(\pi_{q^{*}} \circ \tilde{\varphi}_{q}^{-1}\right)\left(p_{q}\right)\right) \cdot \pi_{q^{*}}^{\prime}\left(\tilde{\varphi}_{q}^{-1}\left(p_{q}\right)\right) \cdot\left(\tilde{\varphi}_{q}^{-1}\right)^{\prime}\left(p_{q}\right) \\
& \underbrace{=}_{\text {(4.1) }} \varphi_{q}^{\prime}(p) \cdot \pi_{q^{*}}^{\prime}\left(p_{q^{*}}\right) \cdot\left(\tilde{\varphi}_{q}^{-1}\right)^{\prime}\left(p_{q}\right) \underbrace{=}_{\varphi_{q}^{\prime}(p)=1} \pi_{q^{*}}^{\prime}\left(p_{q^{*}}\right) \cdot\left(\tilde{\varphi}_{q}^{-1}\right)^{\prime}\left(p_{q}\right) .
\end{aligned}
$$

Hence

$$
\left|\rho_{q^{*}}(p)-\rho_{q}(p)\right|=\frac{1}{\left|\pi_{q^{*}}^{\prime}\left(p_{q^{*}}\right)\right|} \cdot\left|\rho_{\mathbb{D}}\left(p_{q^{*}}\right)-\frac{\rho_{\mathbb{D}}\left(\tilde{\varphi}_{q}\left(p_{q^{*}}\right)\right)}{\left|\left(\tilde{\varphi}_{q}^{-1}\right)^{\prime}\left(p_{q}\right)\right|}\right| \longrightarrow 0 \quad \text { when } \quad q \rightarrow q^{*},
$$

since $\tilde{\varphi}_{q} \rightarrow$ id in the $C^{1}$-norm in a neighbourhood of $p_{q^{*}}$ when $q \rightarrow q^{*}$.

4.2. Estimates of metrics with infinitely many singularities. We are now able to prove Theorem 1.5, which is the key statement for the proof of Theorem 4.1.

Theorem 4.3. Let $K>1$ and let $z_{i} \rightarrow \infty$ be a sequence of points satisfying $\left|z_{i}\right|<\left|z_{i+1}\right| \leq K\left|z_{i}\right|$. Let $\mathcal{O}=\left(\mathbb{C}, \nu_{\mathcal{O}}\right)$, where

$$
\nu_{\mathcal{O}}(z)= \begin{cases}2 & \text { if } z=z_{i} \text { for some } i \\ 1 & \text { otherwise. }\end{cases}
$$

Then the density $\rho_{\mathcal{O}}$ of the hyperbolic metric on $\mathcal{O}$ satisfies

$$
\frac{1}{\rho_{\mathcal{O}}(z)} \leq \mathrm{O}(|z|) \quad \text { as } z \rightarrow \infty \text {. }
$$

Proof. Since for any $a \in \mathbb{C}, z+a=\mathrm{O}(z)$ as $z \rightarrow \infty$, we can assume without loss of generality that 0 is one of the ramified points of $\mathcal{O}$.

Let $z \neq z_{i}$ be an arbitrary but fixed point in $\mathcal{O}$. Depending on $z$, we choose $b=b(z)=z_{k}$, where $z_{k}$ satisfies $\left|z_{k}\right| \geq 2|z|$ and is minimal with this property, i.e., if $\left|z_{j}\right|<\left|z_{k}\right|$, then $\left|z_{j}\right|<2|z|$. It follows immediately that

$$
2|z| \leq|b|=\left|z_{k}\right| \leq K\left|z_{k-1}\right| \leq 2 K|z| \text {. }
$$

Next as we set $c=c(z)=z_{l}$, where $z_{l}$ is minimal with the property $\left|z_{l}\right| \geq 2|b|$. We then obtain

$$
4|z| \leq 2|b| \leq|c|=\left|z_{l}\right| \leq K\left|z_{l-1}\right| \leq 2 K|b| \leq 4 K^{2}|z| .
$$

For any three pairwise distinct points $p, q, r \in \mathbb{C}$ denote by $\mathcal{O}_{p, q, r}:=\left(\mathbb{C}, \nu_{p, q, r}\right)$ the orbifold defined by

$$
\nu_{p, q, r}(w)= \begin{cases}2 & \text { if } w \in\{p, q, r\} \\ 1 & \text { otherwise }\end{cases}
$$




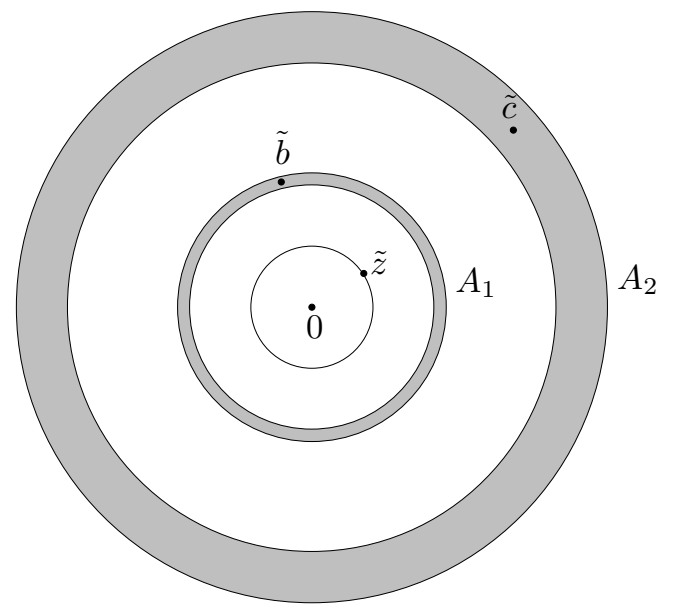

Figure 1 . The parameters $\tilde{b}$ and $\tilde{c}$ belong to the compact annuli $A_{1}$ and $A_{2}$.

Note that every such orbifold is hyperbolic, since its Euler characteristic equals $-1 / 2$. We denote by $\rho_{p, q, r}$ the density of the hyperbolic metric on $\mathcal{O}_{p, q, r}$.

Observe first that $\mathcal{O}$ is holomorphically embedded in $\mathcal{O}_{0, b, c}$, and it follows from Theorem 2.4 that $\rho_{\mathcal{O}}(w)>\rho_{0, b, c}(w)$ holds for all $w \in \mathcal{O}$. Let $\tilde{b}=\tilde{b}(z):=b /|z|$ and $\tilde{c}=\tilde{c}(z):=c /|z|$. Then the map

$$
S_{z}: \mathcal{O}_{0, b, c} \rightarrow \mathcal{O}_{0, \tilde{b}, \tilde{c}}, \quad w \mapsto \frac{w}{|z|}
$$

is obviously a conformal isomorphism and hence an isometry. Altogether, we obtain

$$
\rho_{0, \tilde{b}, \tilde{c}}(S(w))=\rho_{0, b, c}(w) \cdot|w|<\rho_{\mathcal{O}}(w) \cdot|w| .
$$

Let $z:=S(z)=z /|z|$. Then equations (4.2) and (4.3) yield

$$
2 \leq|\tilde{b}| \leq 2 K \quad \text { and } \quad 4 \leq|\tilde{c}| \leq 4 K^{2}
$$

i.e., $\tilde{b} \in A_{1}=A_{1}(K):=\{w: 2 \leq|w| \leq 2 K\}$ and $\tilde{c} \in A_{2}=A_{2}(K):=\{w: 4 \leq$ $\left.|w| \leq 4 K^{2}\right\}$ belong to compact annuli disjoint from $\tilde{z}$ (see Figure 1).

By Theorem 4.2 the map

$$
\left\{(x, y) \in \mathbb{C}^{2}: x, y \notin\{0, \tilde{z}\}, x \neq y\right\} \rightarrow(0, \infty), \quad(x, y) \mapsto \rho_{0, x, y}(\tilde{z})
$$

is continuous as a composition of two continuous maps, hence it attains a minimum (and maximum) on the compact set $A_{1} \times A_{2}$. Furthermore, the density function $\rho_{0, x, y}$ is continuous, and it attains a minimum (and maximum) over the unit circle. Hence there exist constants $0<m(K), M(K)<\infty$ depending only on $K$ such that

$$
m(K)<\rho_{0, \tilde{b}, \tilde{c}}(\tilde{z})<M(K) .
$$

By setting $w=z$ in equation (4.4) we finally get

$$
m(K) \cdot \frac{1}{|z|} \leq \frac{\rho_{0, \tilde{b}, \tilde{c}}(\tilde{z})}{|z|}<\rho_{\mathcal{O}}(z),
$$

and the assertion of the theorem follows.

Theorem 4.3 and Corollary 3.5 immediately imply the following. 
Corollary 4.4. Let $f$ be strongly subhyperbolic and let $\left(\widetilde{\mathcal{O}}_{f}, \mathcal{O}_{f}\right)$ be dynamically associated to $f$. Then

$$
\frac{1}{\tilde{\rho}_{f}(z)} \leq \mathrm{O}(|z|) \quad \text { as } \quad z \rightarrow \infty
$$

where $\tilde{\rho}_{f}(z)$ denotes the density of the hyperbolic metric on $\widetilde{\mathcal{O}}_{f}$.

4.3. Proof of uniformity. Using Theorems 4.3 and 4.2 we can finally deduce that $f: \widetilde{\mathcal{O}}_{f} \rightarrow \mathcal{O}_{f}$ is a uniform expansion with respect to the hyperbolic metric of $\mathcal{O}_{f}$.

Proof of Theorem 4.1. Let $\tilde{\rho}_{f}$ and $\rho_{f}$ denote the densities of the hyperbolic metrics on $\widetilde{\mathcal{O}}_{f}$ and $\mathcal{O}_{f}$, respectively. Since $f: \widetilde{\mathcal{O}}_{f} \rightarrow \mathcal{O}_{f}$ is a covering map, our claim is equivalent to the statement that there is a constant $E>1$ such that

$$
\frac{\tilde{\rho}_{f}(z)}{\rho_{f}(z)} \geq E>1
$$

By Proposition $3.2(a)$, the underlying domains $\widetilde{S}_{f}$ and $S_{f}$ have no common boundary points in $\mathbb{C}$. Hence it only remains to check that for some $E^{\prime}>1$,

$$
\lim _{z \rightarrow \infty} \frac{\tilde{\rho}_{f}(z)}{\rho_{f}(z)} \geq E^{\prime}>1
$$

Let $C \subset \mathcal{O}_{f}$ be the complement of a closed Euclidean disk centred at 0 such that $\nu_{f}(z)=1$ for all $z \in C$, and denote by $\rho_{C}$ the density of the hyperbolic metric on $C$. Then there is a right half-plane $H \subset \mathbb{C}$ such that the map exp : $H \rightarrow C, z \mapsto \mathrm{e}^{z}$ is a covering. Hence the asymptotic behaviour of $\rho_{C}$ is given by

$$
\rho_{C}(z)=\mathrm{O}\left(\frac{1}{|z| \cdot \log |z|}\right) \quad \text { as } \quad z \rightarrow \infty .
$$

By Theorem $2.4 \rho_{C}(z) \geq \rho_{f}(z)$, and so

$$
\rho_{f}(z) \leq \mathrm{O}\left(\frac{1}{|z| \cdot \log |z|}\right) \quad \text { as } \quad z \rightarrow \infty
$$

It now follows from Corollary 4.4 that

$$
\frac{\rho(z)}{\tilde{\rho}(z)} \leq \mathrm{O}\left(\frac{1}{\log |z|}\right)
$$

and hence

$$
\frac{\tilde{\rho}(z)}{\rho(z)} \rightarrow \infty \quad \text { as } \quad z \rightarrow \infty
$$

Remark. We already mentioned in the introduction that if we replace the ramified points by punctures, i.e., if we consider the hyperbolic domain $U:=\mathbb{C} \backslash\left\{z_{j}: j \in \mathbb{N}\right\}$ instead of the orbifold $\mathcal{O}$, the same bound for the asymptotic behaviour of the density map near $\infty$ can be obtained using standard estimates of the hyperbolic metric in the twice-punctured plane [18, Lemma 2.1]. As will become clear in the proof of Proposition 4.6, the orbifold for which the set of ramified points is given by $\{2 k \pi i: k \in \mathbb{Z}\}$ shows that our estimate is best possible. 
4.4. Cosine maps. We say that $F_{a, b}$ is a cosine map if it can be written as

$$
F_{a, b}(z)=a \mathrm{e}^{z}+b \mathrm{e}^{-z}
$$

for some $a, b \in \mathbb{C}^{*}$. Every such function $F_{a, b}$ has exactly two critical values, namely $v_{1}=2 \sqrt{a b}$ and $v_{2}=-2 \sqrt{a b}$. Furthermore, if $z \in \mathbb{C}$ is a preimage of a critical value, then $z$ is a critical point satisfying $\operatorname{deg}\left(F_{a, b}, z\right)=2$, which implies that $v_{1}$ and $v_{2}$ are totally ramified. It is easy to check that $F_{a, b}$ has no asymptotic values, hence the critical values $v_{1}$ and $v_{2}$ are the only singular values. In particular, every subhyperbolic cosine map is automatically strongly subhyperbolic.

In 24] Schleicher studied landing properties of those cosine maps for which the critical values are strictly preperiodic. Note that the Julia set of every such function equals $\mathbb{C}$. He proved that for such a map, every point in $\mathbb{C}$ is either on a dynamic ray or the landing point of a dynamic ray. This result will also follow from Theorem 1.2. Moreover, our proof in the case of strongly subhyperbolic cosine maps is considerably more concise and elementary than the proof of the general statement or the proof of the (weaker) statement [24, Theorem 6]. The reason is that for strongly subhyperbolic cosine maps we can compute explicitly the required estimates of the metrics of certain dynamically associated orbifolds.

Let us start with a simple observation.

Proposition 4.5. Let $F=F_{a, b}$ be strongly subhyperbolic but not hyperbolic. Then there exists a point $p \in P_{\mathcal{J}} \backslash S(F)$.

Proof. Since $F$ is not hyperbolic, it follows that at least one critical value of $F$ belongs to $\mathcal{J}(F)$. If $P_{\mathcal{J}} \subset S(F)$, then $P_{\mathcal{J}}$ is a forward invariant set of size at most two, so each of its elements has period at most two. Each element of $P_{\mathcal{J}}$ is then totally ramified and therefore belongs to a superattracting cycle. This clearly cannot happen since superattracting cycles belong to the Fatou set.

For simplicity, let us assume that $\left\{v_{1}, v_{2}\right\} \subset \mathcal{J}(F)$; the case when $\mathcal{F}(F) \neq \emptyset$ can be treated in a very similar way (and is even easier). Let $\mathcal{O}_{F}=\left(\mathbb{C}, \nu_{F}\right)$ and $\widetilde{\mathcal{O}}_{F}=\left(\mathbb{C}, \tilde{\nu}_{F}\right)$, where

$$
\nu_{F}(w)=\operatorname{lcm}\left\{\operatorname{deg}\left(F^{n}, z\right), \text { where } F^{n}(z)=w\right\} \quad \text { and } \quad \tilde{\nu}_{F}(z)=\frac{\nu(F(z))}{\operatorname{deg}(F, z)} .
$$

It is straightforward to check that $\left(\widetilde{\mathcal{O}}_{F}, \mathcal{O}_{F}\right)$ is a pair of orbifolds dynamically associated to $F$. (In fact, this is how we constructed canonical orbifolds in the proof of Proposition 3.2.) In particular, $\nu_{F}(z) \in\{1,2,4\}$ for all $z \in \mathbb{C}$.

Let us fix a point $p \in P_{\mathcal{J}} \backslash S(F)$. Then $p$ has only regular preimages $p_{i}$, for which necessarily $\tilde{\nu}_{F}\left(p_{i}\right)=\nu_{F}(p) \in\{2,4\}$. Since $F$ is $2 \pi i$-periodic, the orbifold $\widetilde{\mathcal{O}}_{F}$ is holomorphically embedded in the orbifold $\mathcal{O}_{0}=\left(\mathbb{C}, \nu_{0}\right)$ defined by

$$
\nu_{0}(z)= \begin{cases}\nu_{F}(p) & \text { if } w=2 \pi n i \text { for some } n \in \mathbb{Z}, \\ 1 & \text { otherwise. }\end{cases}
$$

In particular, if $\tilde{\rho}_{F}$ and $\rho_{0}$ denote the densities of the hyperbolic metrics on $\widetilde{\mathcal{O}}_{F}$ and $\mathcal{O}_{0}$, respectively, then

$$
\tilde{\rho}_{F}(z)>\rho_{0}(z+\eta)
$$

holds for all $z \in \mathbb{C}$ and some suitable constant $\eta$. For $\rho_{0}$ we can give the following explicit lower bound. 
Proposition 4.6. There exist constants $C, D>0$ such that for all $z \in \mathbb{C}$,

$$
\rho_{0}(z) \geq \frac{1}{C+D|\operatorname{Re}(z)|} .
$$

Proof. For all points in the punctured half-plane $\{z \neq 0: \operatorname{Re}(z) \leq 1 / 2\} \subset \mathbb{C} \backslash\{0,1\}$, the density $\rho_{1}$ of the hyperbolic metric of $\mathbb{C} \backslash\{0,1\}$ can be bounded from below by

$$
\frac{1}{\rho_{1}(z)} \leq C_{1} \cdot|z| \cdot\left(C_{2}+|\log | z||\right),
$$

where $C_{1}:=2 \sqrt{2}$ and $C_{2}:=4+\log (3+2 \sqrt{2}) 2$, p. 476].

Let $\mathcal{O}_{2}:=\left(\mathbb{C}^{*}, \nu_{2}\right)$, where $\nu_{2}(1)=2$ and $\nu_{2}(z)=1$ for all $z \neq 1$. We easily see that the map $p: \mathbb{C} \backslash\{0,1\} \rightarrow \mathcal{O}_{2}, z \mapsto-4\left(z^{2}-z\right)$ is a covering map and hence a local isometry. A simple calculation yields

$$
\frac{1}{\rho_{2}(w)} \leq 2 C_{1} \cdot|\sqrt{1-w}| \cdot|1-\sqrt{1-w}| \cdot\left(C_{2}+\log 2+|\log | 1-\sqrt{1-w}||\right),
$$

where $\rho_{2}(z)$ is the density of the hyperbolic metric of $\mathcal{O}_{2}$ and $\sqrt{z}$ denotes the branch of the square root with nonnegative real part. (Obviously, the same is true for the other branch as well, but we make a choice in order to keep the computation clear.)

Since $\mathcal{O}_{0} \rightarrow \mathcal{O}_{2}, z \mapsto \mathrm{e}^{z}$ is a covering map, it follows that

$$
\frac{1}{\rho_{0}(z)} \leq 2 C_{1} \cdot\left|\sqrt{1-\mathrm{e}^{z}}\right| \cdot\left|1-\sqrt{1-\mathrm{e}^{z}}\right| \cdot\left(C_{2}+\log 2+|\log | 1-\sqrt{1-\mathrm{e}^{z}}||\right) \cdot\left|\mathrm{e}^{-z}\right|
$$

for every $z \in \mathcal{O}_{0}$. Let us simplify the above expression. We note, by expanding with $\left|1+\sqrt{1-\mathrm{e}^{z}}\right|$, that

$$
\frac{\left|\sqrt{1-\mathrm{e}^{z}}\right| \cdot\left|1-\sqrt{1-\mathrm{e}^{z}}\right|}{\left|\mathrm{e}^{z}\right|}=\frac{\left|\sqrt{1-\mathrm{e}^{z}}\right|}{\left|1+\sqrt{1-\mathrm{e}^{z}}\right|},
$$

and this is bounded from above by 1 since $\operatorname{Re}\left(\sqrt{1-\mathrm{e}^{z}}\right)>0$.

Let us now consider $|\log | 1-\sqrt{1-e^{z}}||$. Since $|\log 1 / z|=|\log z|$, it is enough to restrict to the case when $\left|1-\sqrt{1-e^{z}}\right| \geq 1$. So here we get

$$
\begin{aligned}
1 & \leq\left|1-\sqrt{1-\mathrm{e}^{z}}\right| \leq 1+\sqrt{\left|1-\mathrm{e}^{z}\right|} \leq \max \left\{2,2 \sqrt{\left|1-\mathrm{e}^{z}\right|}\right\} \\
& \leq \max \left\{2,2 \sqrt{1+\left|\mathrm{e}^{z}\right|}\right\} \leq \max \left\{2,2\left(1+\left|\mathrm{e}^{z}\right|\right)\right\} \leq 2\left(1+\left|\mathrm{e}^{z}\right|\right) \\
& \leq \max \left\{2,2\left|\mathrm{e}^{z}\right|\right\},
\end{aligned}
$$

and hence

$$
|\log | 1-\sqrt{1-\mathrm{e}^{z}}|| \leq \max \left\{\log 2, \log 2+\log \mathrm{e}^{\operatorname{Re}(z)}\right\} \leq \log 2+|\operatorname{Re}(z)| .
$$

Together, these estimates yield the proof. (More precisely, we see that one can choose $C=2 C_{1}\left(C_{2}+\log 2\right)$ and $D=2 C_{1}$.)

Remark. For arbitrary pairwise distinct points $a, b, c \in \mathbb{C}$ let us denote by $\mathcal{O}_{a, b, c}$ the $\mathbb{C}$-orbifold with signature $(2,2,2)$ with $a, b$ and $c$ being the ramified points. Given an orbifold $\mathcal{O}_{a, b, c}$, the Möbius map $M(z)=\frac{a-b}{2} z+\frac{a+b}{2}$ maps $1,-1$ and $\tilde{z}:=\frac{2 c-a-b}{a-b}$ to $a, b$ and $c$, respectively, and hence is an orbifold covering map from $\mathcal{O}_{1,-1, \tilde{z}}$ to $\mathcal{O}_{a, b, c}$. Let $\mathcal{O}_{0}^{\prime}$ be a $\mathbb{C}$-orbifold whose set of ramified points is a $2 \pi$-periodic sequence of preimages of $\tilde{z}$ under $\cos z$. Then the cosine map is a holomorphic orbifold map from $\mathcal{O}_{0}^{\prime}$ to $\mathcal{O}_{1,-1, \tilde{z}}$. For the hyperbolic metric on $\mathcal{O}_{0}^{\prime}$ we have the same asymptotic behaviour when $|z| \rightarrow \infty$ as for $\mathcal{O}_{0}$, hence this observation enables us to estimate the hyperbolic metric of an arbitrary $\mathbb{C}$-orbifold with signature $(2,2,2)$ using simple 
calculations. This provides an alternative way of proving Theorem 4.3 which though it is less elegant - uses only elementary observations.

\section{Construction of a semiconjugacy}

Recall that our goal is to construct a continuous and surjective map $\phi: \mathcal{J}(g) \rightarrow$ $\mathcal{J}(f)$, where $g$ is any map of disjoint type that belongs to the family

$$
\left\{g_{\lambda}(z)=f(\lambda z): \lambda \in \mathbb{C}\right\}
$$

such that

$$
f \circ \phi(z)=\phi \circ g(z)
$$

holds for all $z \in \mathcal{J}(g)$. Recall that by [18, Theorem 5.2], any two such maps $g$ and $g \prime$ are conjugate on their Julia sets, hence it is enough to prove the statement for one such map. We start with the construction of such a map $g$.

Let us fix a pair of orbifolds $\left(\widetilde{\mathcal{O}}_{f}, \mathcal{O}_{f}\right)$ dynamically associated to $f$ with underlying surfaces $\widetilde{S}_{f}$ and $S_{f}$, respectively. Note that, by Proposition [3.2 $(a), S_{f}$ can be written as $S_{f}=\mathbb{C} \backslash C$, where $C$ is a, possibly empty, compact set.

Observe that for every $\lambda \in \mathbb{C}^{*}, S\left(g_{\lambda}\right)=S(f)$. Let $K>0$ be sufficiently large such that $(P(f) \cup C) \subset\{|z|<K / 2\}$. Since $f$ is entire, it maps bounded sets to bounded sets, hence there exists $L \geq K$ such that

$$
f^{-1}(\{z:|z|>L\}) \subset\{z:|z|>K+1\} .
$$

Let us fix a constant $L \geq K$ with this property and define $\mu:=K / L$. It then follows that if $g=g_{\mu}$ and $z$ is a point with $|g(z)|>L$, then $|\mu z|>K+1$ and hence $|z|>L+L / K$. This means,

$$
g^{-1}(\{z:|z|>L\}) \subset\{z:|z|>L+L / K\},
$$

and, in particular, it follows from Proposition 2.8 that $g$ is of disjoint type.

Define

$$
V_{j}:=f^{-j}(\{z:|z|>K\}) \quad \text { and } \quad U_{j}:=g^{-j}(\{z:|z|>L\}) .
$$

Remark. By construction, $V_{0} \subset S_{f}$, so it follows from Proposition $3.2(f)$ that $V_{j} \subset S_{f} \cap \widetilde{S}_{f}$ for all $j \geq 0$. Furthermore, $V_{0}$ is disjoint from the set of ramified points $B_{f}$ of $\mathcal{O}_{f}$, and since $f\left(B_{f}\right) \subset B_{f}$, it follows that $V_{j} \hookrightarrow \mathcal{O}_{f}$ and $V_{j} \hookrightarrow \widetilde{\mathcal{O}}_{f}$ are holomorphic. Also, $U_{j+1} \subset U_{j}$, since $g$ is of disjoint type. Furthermore, $\mathcal{J}(g)$ is the set of those points that are never mapped into $\mathbb{C} \backslash U_{0}$, hence $\mathcal{J}(g)$ equals the limit of the domains $U_{j}$.

We want to construct a sequence of conformal isomorphisms

$$
\phi_{j}: U_{j-1} \rightarrow V_{j-1}
$$

for $j \geq 1$ and with $\phi_{0} \equiv$ id such that

$$
f \circ \phi_{j+1}=\phi_{j} \circ g .
$$

We will proceed inductively. Since $\phi_{0} \equiv$ id, the map $\phi_{1}$ is given by the formula $\phi_{1}(z)=\mu z$. For a point $z \in V_{0}$ let $\gamma_{1}(z)$ be the straight line segment connecting $z=\phi_{0}(z)$ and $\mu z=\phi_{1}(z)$ (we can actually choose $\gamma_{1}$ to be any rectifiable curve which connects $z$ and $\phi_{1}(z)$ within the domain $V_{0}$ ). To define $\phi_{2}$ at a point $z \in U_{1}$, we consider the line segment $\gamma_{1}(g(z)) \subset V_{0}$. By definition of $V_{j}$,

$$
f^{-1}\left(\gamma_{1}(g(z))\right) \subset V_{1} .
$$




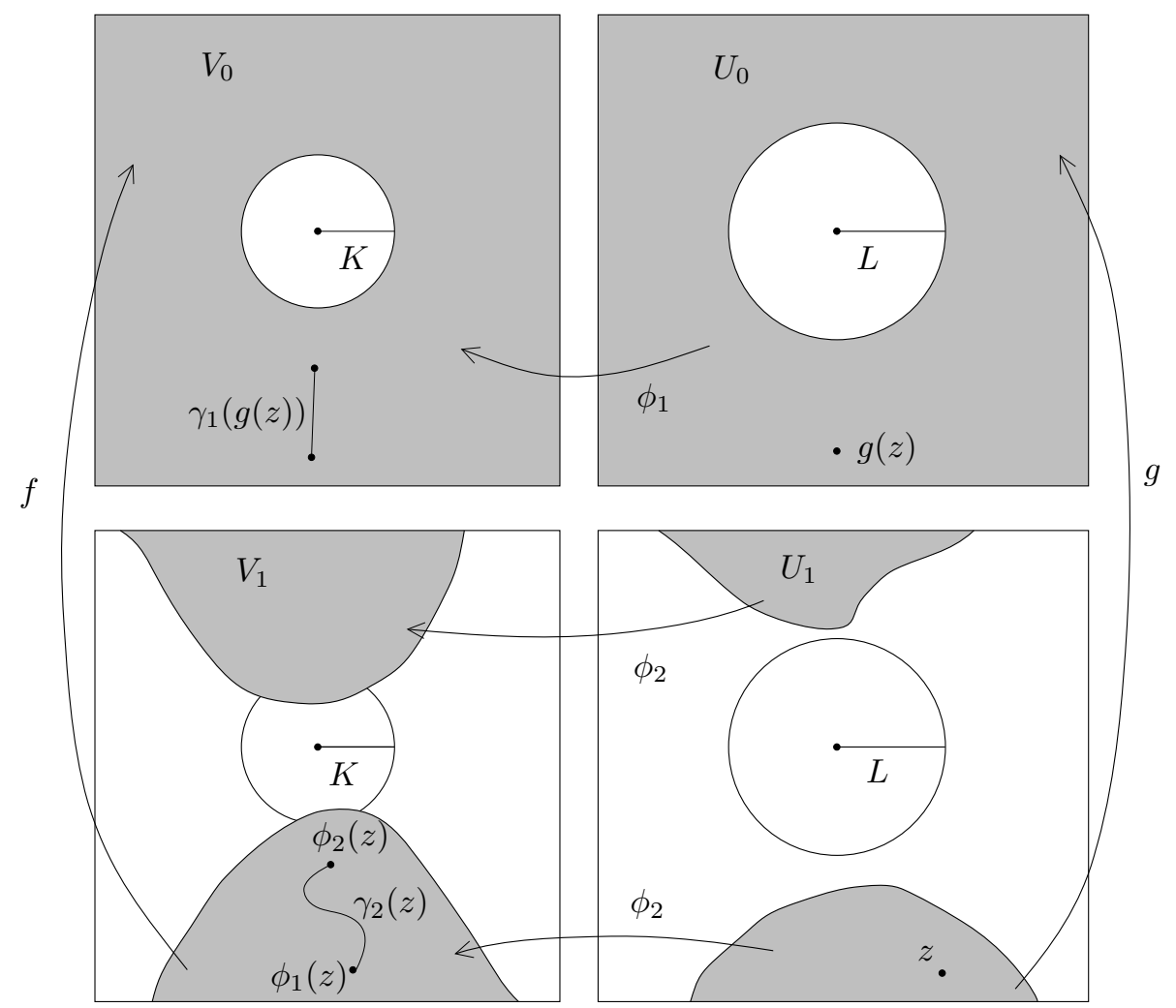

Figure 2. The construction of the curve $\gamma_{2}(z)$ and the isomorphism $\phi_{2}$.

Since $f\left(\phi_{1}(z)\right)=g(z)$, there is a preimage component $\gamma_{2}(z)$ of $\gamma_{1}(g(z))$ such that one endpoint of $\gamma_{2}(z)$ is $\phi_{1}(z)$. We define $\phi_{2}(z)$ to be the other endpoint of $\gamma_{2}(z)$ (see Figure 2).

Continuing inductively, we define the curve $\gamma_{j+1}(z) \subset V_{j}$ to be the pullback of $\gamma_{j}(g(z)) \subset V_{j-1}$ under $f$ with one endpoint at $\phi_{j}(z)$, and we define $\phi_{j+1}(z)$ to be the other endpoint $\gamma_{j+1}(z)$.

We want to give some properties of the maps $\phi_{j}$. Since $f$ and $g$ are holomorphic and in particular continuous, each map $\phi_{j}$ is continuous as well. By induction, it also follows that each map $\phi_{j}$ is injective and surjective. Hence each map $\phi_{j}$ is a conformal isomorphism, mapping a component of $U_{j-1}$ onto a component of $V_{j-1}$.

Theorem 5.1. The maps $\left.\phi_{j}\right|_{\mathcal{J}(g)}$ converge uniformly with respect to the hyperbolic orbifold metric $\rho_{f}(z)|d z|$ on $\mathcal{O}_{f}$ to a continuous surjective function

$$
\phi: \mathcal{J}(g) \rightarrow \mathcal{J}(f)
$$

so that $f \circ \phi=\phi \circ g$. Moreover, $\phi(I(g))=I(f)$ and $\left.\phi\right|_{I(g)}$ is a homeomorphism.

Proof. With respect to the hyperbolic metric on $\mathcal{O}_{f}$, we denote by $d_{f}\left(w_{1}, w_{2}\right)$ the distance between two points $w_{1}, w_{2} \in \mathcal{O}_{f}$, and by $\ell_{f}(\gamma)$ the length of a rectifiable curve $\gamma \subset \mathcal{O}_{f}$. Let $z \in U_{j}$. Since $U_{j} \subset U_{j-1}$, both $\phi_{j}$ and $\phi_{j+1}$ are defined in a 
neighbourhood of $z$ and it follows from our construction that

$$
d_{f}\left(\phi_{j+1}(z), \phi_{j}(z)\right) \leq \ell_{f}\left(\gamma_{j+1}(z)\right) .
$$

Since for every point $z \in U_{0}$,

$$
\gamma_{1}(z) \subset\left(\mathbb{C} \backslash \overline{D_{K}(0)}\right) \subset\left(\mathbb{C} \backslash \overline{D_{\frac{K}{2}}(0)}\right) \subset \mathcal{O}_{f},
$$

we obtain an upper bound for $\ell_{f}\left(\gamma_{1}\right)$ by computing its length with respect to the hyperbolic metric in $\mathbb{C} \backslash \overline{D_{\frac{K}{2}}(0)}$, which is given by $|d z| /(|z|(\log |z|-\log (K / 2)))$. Hence

$$
\ell_{f}\left(\gamma_{1}(z)\right) \leq \log \left(\frac{\log |1 / \mu|}{\log |z|-\log (K / 2)}+1\right) \leq \log \left(\frac{\log |1+\mu|}{\log 2}+1\right)=: \alpha .
$$

Recall that by Theorem 4.1, there is a constant $E>1$ such that $\|D f(z)\|_{\mathcal{O}_{f}} \geq E$ holds for all $z \in \widetilde{\mathcal{O}}_{f}$. Since $\gamma_{j+1}(z) \subset V_{j} \subset \widetilde{\mathcal{O}}_{f}$ is obtained as a pullback of $\gamma_{1}\left(g^{j}(z)\right)$ under the map $f^{j}$, it follows from equation (5.1) that

$$
d_{f}\left(\phi_{j+1}(z), \phi_{j}(z)\right) \leq \frac{\alpha}{E^{j}} .
$$

This means that the maps $\left.\phi_{j}\right|_{\mathcal{J}(g)}$ form a Cauchy sequence, and since the orbifold metric is complete, there is a continuous limit function

$$
\phi: \mathcal{J}(g) \rightarrow \mathcal{O}_{f}
$$

Note that $\phi$ necessarily satisfies

$$
d_{f}(\phi(z), z) \leq \sum_{j=0}^{\infty} d_{f}\left(\phi_{j+1}(z), \phi_{j}(z)\right) \leq \sum_{j=0}^{\infty} \alpha \cdot \frac{1}{E^{j}}=\alpha \cdot \frac{E}{E-1}
$$

as well as

$$
f^{n}(\phi(z))=\phi\left(g^{n}(z)\right)
$$

for all $n \in \mathbb{N}$ and all $z \in \mathcal{J}(g)$.

We want to derive some properties of the limit function $\phi$. By equation (5.2),

$$
\phi\left(z_{n}\right) \rightarrow \infty \text { if and only if } z_{n} \rightarrow \infty,
$$

so together with equation (5.3) this implies that $\phi(I(g)) \subset I(f)$. In particular, it follows that $\phi(\mathcal{J}(g)) \subset \mathcal{J}(f)$, since $\mathcal{J}(g)=\overline{I(g)}$ and $\mathcal{J}(f)=\overline{I(f)}$ [ . Now let $w \in I(f)$. Then $w \in V_{j}$ for all sufficiently large $j$, so we can consider the sequence $z_{j}:=\phi_{j}^{-1}(w)$. If $z$ is an accumulation point of $\left(z_{j}\right)$, then $\phi(z)=w$. (Note that by relation (5.4), $z \neq \infty$.) Hence $\phi: I(g) \rightarrow I(f)$ is surjective.

Before we show that $\left.\phi\right|_{I(g)}$ is injective, let us recall that $U_{1}=g^{-1}(\{z:|z|>L\})$ is a countable collection of tracts on which $g$ acts as a covering map. Now let $\alpha \subset$ $U_{0} \backslash U_{1}$ be a curve connecting $\{z:|z|=L\}$ with $\infty$. As in the proof of Proposition 2.9. every component of $g^{-1}\left(U_{0} \backslash \alpha\right)$ is a fundamental domain on which $g$ restricts as a conformal map. Now let $z, \tilde{z} \in I(g)$ be two points such that $\phi(z)=\phi(\tilde{z})=: w$. By definition, $\phi_{j}(z), \phi_{j}(\tilde{z}) \rightarrow w$, and it follows from the inductive definition of the maps $\phi_{j}$ that for every sufficiently large $j$, there exists a fundamental domain $F_{j}$ such that $g^{j}(z), g^{j}(\tilde{z}) \in F_{j}$. On the other hand, it follows from equation (5.3) that $\phi\left(g^{j}(z)\right)=\phi\left(g^{j}(\tilde{z})\right)$ holds for all $j \in \mathbb{N}$. Furthermore, equation (5.2) implies that

$$
d_{f}\left(g^{j}(z), g^{j}(\tilde{z})\right) \leq d_{f}\left(g^{j}(z), \phi\left(g^{j}(z)\right)\right)+d_{f}\left(\phi\left(g^{j}(\tilde{z})\right), g^{j}(\tilde{z})\right) \leq 2 \alpha \cdot \frac{E}{E-1} .
$$


By standard expansion estimates (see e.g. [18, Lemma 2.7]), the distance between $g^{j}(z)$ and $g^{j}(\tilde{z})$ must be unbounded, unless the points $z$ and $\tilde{z}$ are equal, implying that $\phi$ is injective.

Observe that by equation (5.4), $\phi$ can be extended continuously to a map $\widehat{\phi}$ : $\mathcal{J}(g) \cup\{\infty\} \rightarrow \mathcal{J}(f) \cup\{\infty\}$ with $\widehat{\phi}(\infty)=\infty$. The set $\widehat{\phi}(\mathcal{J}(g) \cup\{\infty\})$ is compact since it is the continuous image of a compact set. Furthermore, $\widehat{\phi}(\mathcal{J}(g))=\phi(\mathcal{J}(g))$ is necessarily closed. So

$$
I(f)=\phi(I(g)) \subset \phi(\mathcal{J}(g)) \subset \mathcal{J}(f)=\overline{I(f)},
$$

and as $\phi(\mathcal{J}(g))$ is closed, it follows that $\phi(\mathcal{J}(g))=\mathcal{J}(f)$, hence $\phi$ is surjective.

Since the restriction of the map $\phi$ in Theorem 5.1 to the escaping set of the disjoint type map is a homeomorphism, we obtain the following result as an immediate consequence of Theorem 5.1 and Proposition 2.9.

Corollary 5.2. The escaping set of a strongly subhyperbolic map is not connected.

Remark. Dierk Schleicher kindly pointed out that the escaping set of the cosine map $z \mapsto \pi \sinh z$ mentioned in the introduction is obviously disconnected: the imaginary axis consists of points with bounded orbits and it disconnects the escaping set (for details on this special function, see Appendix A).

To state our next corollary, we need to introduce the notion of a dynamic ray.

Definition 5.3 (Dynamic rays and ray tails). A ray tail of a transcendental entire map $f$ is an injective curve

$$
g:\left[t_{0}, \infty\right) \rightarrow I(f)
$$

(where $t_{0}>0$ ) such that for each $n \in \mathbb{N}, \lim _{t \rightarrow \infty} f^{n}(g(t))=\infty$ and such that, as $n \rightarrow \infty, f^{n}(g(t)) \rightarrow \infty$ uniformly in $t$.

A dynamic ray of $f$ is then a maximal injective curve $g:(0, \infty) \rightarrow I(f)$ such that $\left.g\right|_{\left[t_{0}, \infty\right)}$ is a ray tail for every $t_{0}>0$.

In terms of dynamic rays, our main result implies the following topological description of the Julia set of certain strongly subhyperbolic maps.

Corollary 5.4. Let $f=f_{1} \circ \cdots \circ f_{n}$ be a strongly subhyperbolic map where every $f_{i}$ has finite order and a bounded set of singular values.

Then $\mathcal{J}(f)$ is a pinched Cantor bouquet consisting of dynamic rays of $f$ and their endpoints. In particular, all dynamic rays of $f$ land and every point in $\mathcal{J}(f)$ is either on a dynamic ray or the landing point of a dynamic ray of $f$.

Recall that by a pinched Cantor bouquet we mean a quotient of a Cantor bouquet by a closed equivalence relation on its endpoints. Note that Corollary 5.4 implies Corollary 1.3 from the introduction.

Proof. Let $g$ and $\phi$ be as in Theorem 5.1. Then $\mathcal{J}(g)$ is a Cantor bouquet consisting of dynamic rays of $g$ and their endpoints [22, p. 15, Theorem 4.7]. Since $\phi$ is surjective, $\mathcal{J}(f)$ is a pinched Cantor bouquet consisting of dynamic rays of $f$ and their endpoints.

Remark. In 22 the authors only remark but do not explicitly prove that the Julia set of a disjoint type map which can be written as a finite composition of finite order functions is a Cantor bouquet. However, this can be deduced, for instance, 
using a topological characterization such as the one given in [1, Theorem 3.11]. We will not state the details here since it would require a lot of vocabulary from point-set topology, which would be of no further use in this article.

If $f$ is a function as in Corollary 5.4 then one can encode the "pinching" of the Cantor bouquet (which equals $\mathcal{J}(f)$ ) combinatorially using itineraries. We will elaborate this explicitly in the case of the map $z \mapsto \pi \sinh z$ in the following section. However, such a concept can be developed in the more general setting of Corollary 5.4 this is contained in the author's thesis [12, Chapter 5]. For more information on itineraries of exponential and cosine maps, see e.g. 23, 24.

\section{Appendix A: Model of the dynamics of a map $f$ with $\mathcal{J}(f)=\mathbb{C}$}

This section is dedicated to the description of the topological dynamics of the function

$$
f(z):=\pi \sinh z .
$$

We will define a "simple" model consisting of a topological space $\bar{X}$ and a map $\mathcal{M}: \bar{X} \rightarrow \bar{X}$ such that if $g$ is any map of disjoint type in the family $g_{\lambda}: z \mapsto \lambda \sinh z$, then

- $\mathcal{J}(g)$ is homeomorphic to $\bar{X}$, and

- $\left.\mathcal{M}\right|_{\bar{X}}$ is conjugate to $\left.g\right|_{\mathcal{J}(g)}$.

We will transfer the ideas from [17, where such a model was constructed for exponential maps whose singular value belongs to some attracting basin. The adaptation of [17 to the maps we are interested in is particularly simple since in left and right half-planes, sufficiently far away from the imaginary axis, any map $g_{\lambda}$ with $\lambda>0$ is essentially the same (i.e., up to a constant factor) as $z \mapsto \mathrm{e}^{-z}$ and $z \mapsto \mathrm{e}^{z}$, respectively. For this reason, we will skip the details and refer, for further consideration, to [17] as well as the extensive work on dynamics of cosine maps by Rottenfußer and Schleicher [21].

Once we have constructed such a model for a disjoint type map $g \in\left\{g_{\lambda}\right\}$, Theorem [5.1 tells us that there is a semiconjugacy between $g$ and $f$ on their Julia sets, and hence also between the model map $\mathcal{M}$ and $f$. The combinatorial dynamics of $f$ on $\mathcal{J}(f)$ was already established in [24, 25] and we will summarize the required results here.

Dynamics within the one-parameter family. Let us consider the family that consists of the maps $g_{\lambda}(z):=\lambda \sinh z$ with $\lambda>0$ (hence $f=g_{\pi}$ ). The critical values of $g_{\lambda}$ are $\pm \lambda i$. Every map $g_{\lambda}: \mathbb{R} \rightarrow \mathbb{R}$ is a homeomorphism with $g_{\lambda}(0)=0$ and $\mathbb{R} \backslash\{0\} \subset I\left(g_{\lambda}\right)$. Furthermore, $g_{\lambda}(i \mathbb{R}) \subset[-\lambda i, \lambda i]$.

Both critical values of $f$ are mapped by $f$ to the repelling fixed point 0 . Since $f$ (as well as every other $g_{\lambda}$ ) has no asymptotic values, the postsingular set of $f$ equals $\{ \pm \pi i, 0\}$. Hence $f$ is postsingularly finite and strongly subhyperbolic. Furthermore, $\mathcal{J}(f)=\mathbb{C}$.

For $\lambda>0$ chosen sufficiently small, the origin is an attracting fixed point and the subinterval $[-\lambda i, \lambda i]$ of the imaginary axis is mapped into itself and thus belongs to the immediate basin of attraction of 0 . Hence by choosing $\lambda$ sufficiently small, we obtain a map $g_{\lambda}$ of disjoint type (see Proposition 2.8). From now on, we will fix $\lambda_{0}>0$ such that the corresponding map $g_{\lambda_{0}}=: g$ is of disjoint type. 
Note that for every $n \in \mathbb{Z}$, the horizontal line

$$
L_{n}:=\{z: \operatorname{Im} z=(n+1 / 2) \pi\}
$$

is mapped by $g$ to $i \mathbb{R} \backslash\left[-\lambda_{0} i, \lambda_{0} i\right]$. Hence every point $z \in \mathcal{J}(g)$ is contained in one of the horizontal half-strips

$$
\begin{aligned}
& S_{n_{L}}:=\{z: \operatorname{Re} z<0, \operatorname{Im} z \in((n-1 / 2) \pi,(n+1 / 2) \pi)\} \text { or } \\
& S_{n_{R}}:=\{z: \operatorname{Re} z>0, \operatorname{Im} z \in((n-1 / 2) \pi,(n+1 / 2) \pi)\} .
\end{aligned}
$$

Note that the restriction of $g$ to any of the half-strips is a conformal isomorphism onto its image which is the left or right half-plane. (Similar observations hold for any other $g_{\lambda}$ with $\lambda \in \mathbb{R}$.)

Topological model. Let us denote by $\mathbb{Z}_{L}:=\left\{\ldots,-1_{L}, 0_{L}, 1_{L}, \ldots\right\}$ and $\mathbb{Z}_{R}:=$ $\left\{\ldots,-1_{R}, 0_{R}, 1_{R}, \ldots\right\}$ two disjoint copies of $\mathbb{Z}$, and let $\mathscr{S}^{\mathbb{N}}:=\left(\mathbb{Z}_{L} \cup \mathbb{Z}_{R}\right)^{\mathbb{N}}$ be the space of infinite sequences of elements in $\mathbb{Z}_{L} \cup \mathbb{Z}_{R}$. By the previous argument, we can assign to a point $z \in \mathcal{J}(g)$ a unique sequence $\underline{s}=s_{0} s_{1} \ldots \in \mathscr{S}^{\mathbb{N}}$ defined by $g^{n}(z) \in S_{s_{n}}$. We will call such a sequence the external address of $z$. For every $i \in \mathbb{Z}$ we define $\left|i_{L}\right|:=|i|=:\left|i_{R}\right|$. Furthermore, since $\mathcal{J}(g)$ consists of (asymptotically horizontal) dynamic rays and their endpoints [21, Theorem 4.1], our model $\bar{X}$ should be a subset of the space

$$
\mathscr{S}^{\mathbb{N}} \times[0, \infty)
$$

Note that the relation $\ldots i_{L}<i_{R}<(i+1)_{L}<\ldots$ defines an order on $\mathscr{S}^{\mathbb{N}}$. Thus $\mathscr{S}^{\mathbb{N}} \times[0, \infty)$ is equipped with the product of the topology on $\mathscr{S}^{\mathbb{N}}$ (induced by the order relation) and the standard topology on $\mathbb{R}$.

Let $(\underline{s}, t)$ be a point in $\mathscr{S}^{\mathbb{N}} \times[0, \infty)$. We should think of the first entry $s_{0}$ in $\underline{s}$ as the imaginary part of the point (or its height corresponding to our horizontal strips), together with the information as to whether it is lying left or right from the imaginary axis. The second entry $t$ should be thought of as the absolute value of the real part of the point. Hence it is helpful to think of a point $(\underline{s}, t) \in \mathscr{S}^{\mathbb{N}} \times[0, \infty)$ in its "complexified" version $C(\underline{s}, t):=t+\pi i s_{0}$. Let us denote by $T(\underline{s}, t):=t$ the projection onto the second coordinate. We can now define our model map to be

$$
\mathcal{M}: \mathscr{S}^{\mathbb{N}} \times[0, \infty) \rightarrow \mathscr{S}^{\mathbb{N}} \times[0, \infty),(\underline{s}, t) \mapsto\left(\sigma(\underline{s}), F(t)-\pi\left|s_{1}\right|\right),
$$

where $\sigma$ denotes the one-sided shift map and $F(t):=\mathrm{e}^{t}-1$ denotes the standard model map for exponential growth.

Recall that the maps we consider behave like the exponential in each of the halfplanes. The essential characteristic of our model map now is that as for exponential maps, where the size of the image $|C(\mathcal{M}(\underline{s}, t))|$ of a point $(\underline{s}, t)$ is roughly the exponential of its real part. More precisely, $F(t) / \sqrt{2} \leq|C(\mathcal{M}(\underline{s}, t))| \leq F(t)$ whenever $T(\underline{s}, t) \geq 0$. Hence we define the model sets $\bar{X}$ and $X$ to be

$$
\begin{aligned}
& \bar{X}:=\left\{(\underline{s}, t) \in \mathscr{S}^{\mathbb{N}} \times[0, \infty): T\left(\mathcal{M}^{n}(\underline{s}, t)\right) \geq 0 \text { for all } n \geq 0\right\} \text { and } \\
& X:=\left\{(\underline{s}, t) \in \bar{X}: T\left(\mathcal{M}^{n}(\underline{s}, t)\right) \rightarrow \infty \text { as } n \rightarrow \infty\right\} .
\end{aligned}
$$

By [17, Observation 3.1], $\bar{X}$ is homeomorphic to a straight brush. In particular, for every external address $\underline{s}$ there exists a unique $t_{\underline{s}} \in[0, \infty]$ such that $\{t \geq 0$ : $(\underline{s}, t) \in \bar{X}\}=\left[t_{\underline{s}}, \infty\right)$. We denote by $E(\bar{X}):=\left\{\left(\underline{s}, t_{\underline{s}}\right)\right\}$ the set of endpoints of $\bar{X}$.

By iterating forwards under the model map $\mathcal{M}$ and backwards under $g$, we obtain a sequence of maps that converges to a homeomorphism $\Phi: \bar{X} \rightarrow \mathcal{J}(g)$ such that

$$
\Phi \circ \mathcal{M}(z)=g \circ \Phi(z)
$$


for all $z \in \bar{X}$. The key argument for such a limit to exist is again a uniform hyperbolic contraction of the map $g$ and the fact that the mapping behaviour of the model map reflects that of $g$. (For a precise statement see [17, Section 3] or [21. Proposition 3.3].) A proof of the above statement is essentially the same as in the case of exponential maps in [17, Theorem 9.1], which is why we skip the details here. A proof can also be derived by essentially the same estimates as given in the proof of Theorem 5.1 .

By Theorem 5.1 there is a surjective map $\phi: \mathcal{J}(g) \rightarrow \mathcal{J}(f)$ such that $f(\phi(z))=$ $\phi(g(z))$ holds for all $z \in \mathcal{J}(g)$. Moreover, $\phi$ restricts to a homeomorphism between $I(g)$ and $I(f)$. As already mentioned, every point $z \in I(f)$ escapes within the strips $S_{s_{i}}$ with $s_{i} \in \mathbb{Z}_{L} \cup \mathbb{Z}_{R}$, since the forward orbit of any point in the boundary of the strips is bounded. Recall from the proof of Theorem 5.1 that by choosing the inverse branches of the maps $f^{n}$ appropriately, the conjugacy $\phi$ relates the escaping points of $g$ and $f$ with respect to the combinatorics in terms of their external addresses. From Corollary [5.4, we obtain that $\mathcal{M}$ projects to a function $\widetilde{\mathcal{M}}$ on $\widetilde{X}:=\bar{X} / \sim_{p}$, where $\sim_{p}$ is an equivalence relation on the set $E(\bar{X})$ of endpoints of $\bar{X}$ such that $\widetilde{\mathcal{M}}: \widetilde{X} \rightarrow \widetilde{X}$ is conjugate to $f: \mathcal{J}(f) \rightarrow \mathcal{J}(f)$. The equivalence relation $\sim_{p}$ tells us which dynamic rays are being "pinched". We will now describe $\sim_{p}$ explicitly using the results from [24, 25].

Combinatorial description. For every $n \in \mathbb{Z}$ we set

$$
\begin{aligned}
& U_{(n, 0)}:=\{z: \operatorname{Re} z<0, \operatorname{Im} z \in(2 n \pi, 2(n+1) \pi)\} \text { and } \\
& U_{(n, 1)}:=\{z: \operatorname{Re} z>0, \operatorname{Im} z \in(2 n \pi, 2(n+1) \pi)\} .
\end{aligned}
$$

One can easily see that the restrictions $f: U_{(n, 0)} \rightarrow \mathbb{C} \backslash\left(\mathbb{R}^{+} \cup[-\pi i, \pi i]\right)$ and $f: U_{(n, 1)} \rightarrow \mathbb{C} \backslash\left(\mathbb{R}^{-} \cup[-\pi i, \pi i]\right)$ are conformal isomorphisms. We will call a sequence $\left(n_{0}, k_{0}\right)\left(n_{1}, k_{1}\right) \ldots \in(\mathbb{Z} \times\{0,1\})^{\mathbb{N}}$ an itinerary. If $\gamma$ is a dynamic ray such that for every $i \geq 0$ there exists a domain $U_{\left(n_{i}, k_{i}\right)}$ with $f^{i}(\gamma) \subset U_{\left(n_{i}, k_{i}\right)}$, then we assign to $\gamma$ the (well-defined) itinerary $\operatorname{itin}(\gamma)=\left(n_{0}, k_{0}\right)\left(n_{1}, k_{1}\right) \ldots$. Since a dynamic ray of $f$ is either contained in some half-strip $U_{(n, k)}$ or is completely contained in the boundary of such a domain, it follows that an itinerary cannot be assigned to a ray $\gamma$ if and only if there is $n \geq 0$ such that $f^{n}(\gamma)$ equals $\mathbb{R}^{+}$or $\mathbb{R}^{-}$, or equivalently, if $s_{n+j} \equiv 0_{R}$ or $0_{L}$ for all $j \geq 0$, where $\underline{s}=s_{0} s_{1} \ldots$ is the external address of $\gamma$. This means that to every external address $\underline{s}$ in

$$
\mathscr{S}_{+}^{\mathbb{N}}:=\left\{\underline{s}: t_{\underline{s}}<\infty\right\} \backslash\left\{\underline{s}: s_{n+j} \equiv 0_{R} \text { or } 0_{L} \text { for some } n \geq 0 \text { and all } j \geq 0\right\}
$$

we can assign a unique itinerary $\operatorname{itin}(\underline{s}):=\operatorname{itin}\left(\gamma_{\underline{s}}\right)$. Let us first comment on those external addresses that belong to

$$
\mathscr{S}_{-}^{\mathbb{N}}:=\left\{\underline{s}: t_{\underline{s}}<\infty\right\} \backslash \mathscr{S}_{+}^{\mathbb{N}} .
$$

The mapping behaviour of the map $f$ is fairly simple and allows us to describe completely all tuples and quadruples of external addresses in $\mathscr{S}_{-}^{\mathbb{N}}$ for which the respective dynamic rays land together. For instance, for all addresses $\underline{s}^{i}$ that belong to either the left or right quadruple

$$
s_{0} \ldots s_{j}\left\{\begin{array} { c c c } 
{ ( 2 m ) _ { R } } & { ( 2 n + 1 ) _ { R } } & { 1 _ { L } \overline { 0 _ { R } } } \\
{ ( 2 m ) _ { R } } & { ( 2 n ) _ { R } } & { 1 _ { R } \overline { 0 _ { L } } } \\
{ ( 2 m + 1 ) _ { R } ( 2 n + 1 ) _ { L } } & { 1 _ { R } \overline { 0 _ { L } } } \\
{ ( 2 m + 1 ) _ { R } } & { ( 2 n ) _ { L } } & { 1 _ { L } \overline { 0 _ { R } } }
\end{array} \quad s _ { 0 } \ldots s _ { j } \left\{\begin{array}{ccc}
(2 m+1)_{L}(2 n+1)_{R} 1_{L} \overline{0_{R}} \\
(2 m+1)_{L} & (2 n)_{R} & 1_{R} \overline{0_{L}} \\
(2 m)_{L} & (2 n+1)_{L} & 1_{R} \overline{0_{L}} \\
(2 m)_{L} & (2 n)_{L} & 1_{L} \overline{0_{R}}
\end{array}\right.\right.
$$




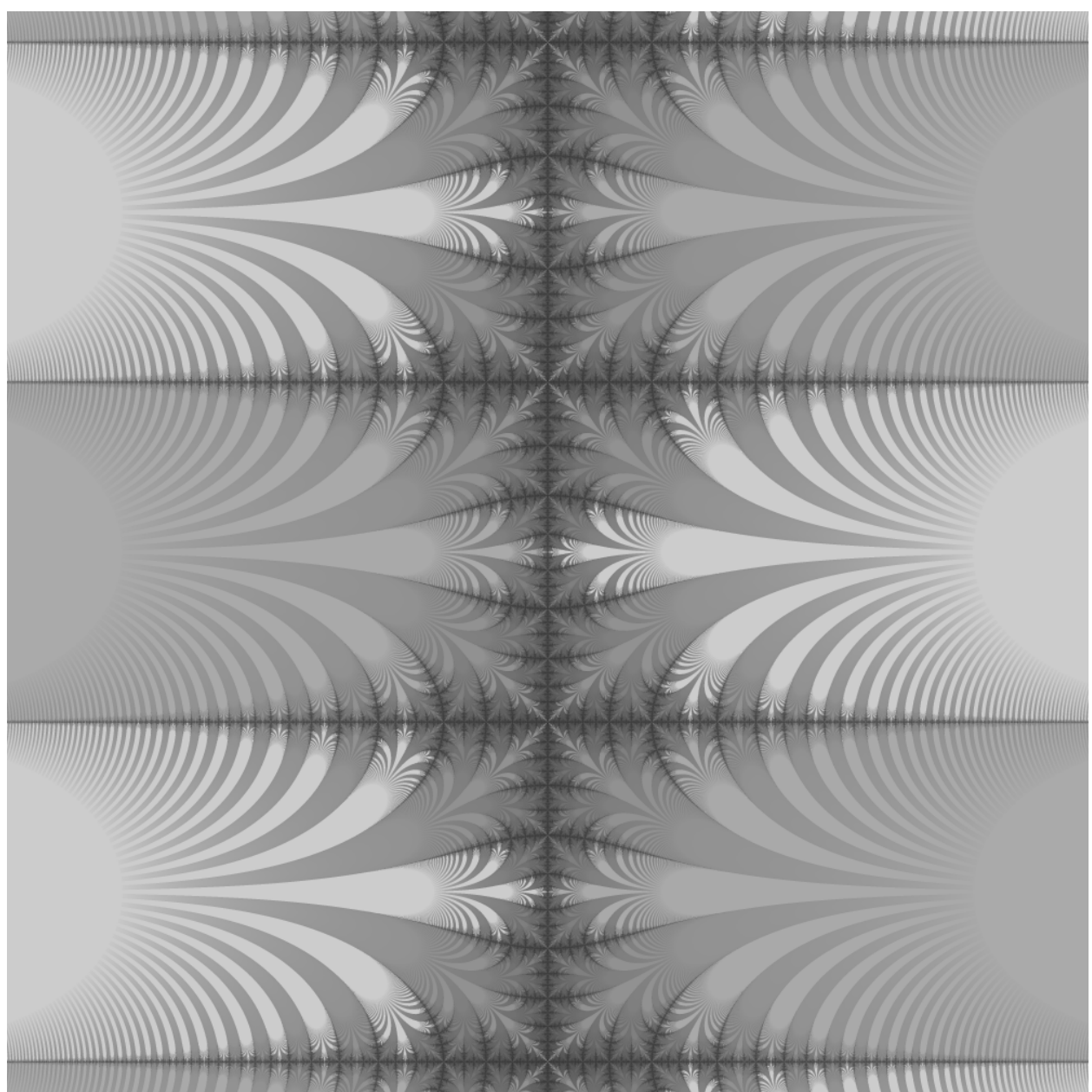

Figure 3 . The Julia set of the map $f(z)=\pi \sinh z$, showing its structure of a pinched Cantor bouquet. This picture was kindly provided by Arnaud Chéritat.

where $m \in \mathbb{Z}$ and $n \geq 0$ are fixed, we define $\left(\underline{s}^{i}, t_{s^{i}}\right) \sim_{p}\left(\underline{s}^{j}, t_{s^{j}}\right)$. We will not list the remaining combinations since there are not so many of them, and each one is easy to determine using elementary computations.

The remaining task is to determine those external addresses in $\mathscr{S}_{+}^{\mathbb{N}}$ such that the corresponding dynamic rays land together. Let $\gamma$ be a dynamic ray with external address $\underline{s} \in \mathscr{S}_{+}^{\mathbb{N}}$ and let $w$ be its landing point. Then either $g$ is the only dynamic ray that lands at $w$ or there is exactly one more such dynamic ray [25]. The latter case occurs if and only if $w$ is eventually mapped into $[\pi i,-\pi i]$ (and remains there without ever being mapped to 0 ). Let $\underline{s}, \underline{s}$ be the external addresses of two dynamic rays landing at the same point $w$. It follows that $\operatorname{itin}(\underline{s})$ and $\operatorname{itin}(\underline{\tilde{s}})$ must be of the 
form

$$
\left(n_{0}, k_{0}\right) \ldots\left(n_{j}, k_{j}\right)\left\{\begin{array}{l}
\left(n_{j+1}, k_{j+1}\right)\left(0, k_{j+2}\right)\left(0, k_{j+3}\right) \ldots \\
\left(n_{j+1}, 1-k_{j+1}\right)\left(0,1-k_{j+2}\right)\left(0,1-k_{j+3}\right) \ldots
\end{array}\right.
$$

or with -1 instead of 0 .

On the other hand, it follows from [24, Lemma 5] and elementary computations that two dynamic rays with itineraries as in equation (5.5) do indeed land together: such dynamic rays have a forward image that lands in the interval $[-\pi i, \pi i]$ and its landing point is never mapped to 0 . So let $\underline{s}, \underline{\tilde{s}} \in \mathscr{S}_{+}^{\mathbb{N}}$. It follows that $\left(\underline{s}, t_{\underline{s}}\right) \sim_{p}\left(\underline{\tilde{s}}, t_{\underline{s}}\right)$ if and only if $\operatorname{itin}(\underline{s})$ and $\operatorname{itin}(\underline{\tilde{s}})$ are of the form given by equation (5.5) (or with -1 instead of 0 ).

Remark. One can certainly relate the model $(\bar{X}, \mathcal{M})$ directly to $\mathcal{J}(f)$. The reason to incorporate a disjoint type map is simply to show what to do when the considered strongly subhyperbolic map $f$ can be embedded in a family where the topological dynamics of disjoint type maps is well understood.

\section{APPEndix B: SubHYPERBOLIC MAPS Without DYNAMICALLY ASSOCIATED ORBIFOLDS}

We want to give an explicit example of a map $\Phi$ that is subhyperbolic, has no asymptotic values, but such that the local degree at points in $\mathcal{J}(\Phi)$ is unbounded.

Let $p$ be a complex polynomial of degree $d \geq 2$ and let $z_{0}$ be a repelling fixed point of $p$ with multiplier $\mu$. By Poincaré's Theorem [16, 28, there exists an entire map $\Phi$, which is called a Poincaré function of $p$ at $z_{0}$, such that the functional equation

$$
\Phi(\mu \cdot z)=p(\Phi(z))
$$

is satisfied for all $z \in \mathbb{C}$. Now let

$$
p(z)=z^{2}-1
$$

and let $\Phi_{0}$ denote a Poincaré function of $p$ at the point $z_{0}:=(1+\sqrt{5}) / 2$. The unique finite critical point of $p$ is 0 . Since $p(0)=-1$ and $p(-1)=0$, the cycle $\{0,-1\}$ is superattracting and, in particular, $P(p) \cap \mathbb{C}=\{0,-1\}$. Note that $p$ has no other attracting or parabolic cycles in $\mathbb{C}$, since every such cycle attracts at least one critical point of $p$ [14, Theorem 8.6]. Also observe that $p$ has no exceptional values (points with a finite backward orbit) in $\mathbb{C}$; it is now not hard to check that $C\left(\Phi_{0}\right)=P(p)=\{0,-1\}$ (see e.g. [5, Theorem 2.10]). It also follows from [13, Proposition 4.3] that $\Phi_{0}$ has no asymptotic values. This is due to the fact that $z_{0}$ does not belong to the boundary of an immediate attracting basin of $p$.

Since 0 is a critical value of $\Phi_{0}$, there exists a point $\tilde{z}$ such that

$$
\Phi_{0}(\tilde{z})=0 \quad \text { and } \quad \Phi_{0}^{\prime}(\tilde{z})=0 .
$$

Let $z_{n}:=\lambda^{n} \tilde{z}$. Using the functional equation (5.6), it is not difficult to see that for every $n \in \mathbb{N}$,

$$
\left.\frac{d^{k}}{d z^{k}} \Phi_{0}(z)\right|_{z=z_{n}}=0 \quad \text { for all } 0 \leq k \leq n
$$

hence for every $n \in \mathbb{N}$, we have $\operatorname{deg}\left(\Phi_{0}, z_{n}\right) \geq n$.

Let us also observe that the critical value 0 has a regular preimage: Since $\Phi_{0}$ has no omitted values, there exists a point $w$ such that $\Phi_{0}(w)=1$. By equation (5.6), 
the point $a:=\lambda w$ is mapped by $\Phi_{0}$ to 0 , and by deriving the functions in equation (5.6), it follows that $\Phi_{0}^{\prime}(a)=2 \Phi_{0}^{\prime}(w) / \lambda \neq 0$.

Let $b$ be a preimage of -1 , chosen sufficiently large such that $|a-b| \cdot\left|\Phi_{0}^{\prime}(a)\right|>1$. Now consider the map

$$
\Phi(z):=(a-b) \cdot \Phi_{0}(z)+a .
$$

Note that $A(\Phi)=\emptyset$ since $\Phi$ and $\Phi_{0}$ differ only by postcomposition with a conformal map. It also follows immediately that $C(\Phi)=\{a, b\}, \Phi(a)=a$ and $\Phi(b)=b$, hence $\Phi$ is postsingularly finite and in particular subhyperbolic. Moreover, since $\left|\Phi^{\prime}(a)\right|=|a-b| \cdot\left|\Phi_{0}^{\prime}(a)\right|>1$, the critical value $a$ is a repelling fixed point of $\Phi$ and hence belongs to $\mathcal{J}(\Phi)$, implying that $\Phi$ is not hyperbolic. Finally note that the points $z_{n}$ are mapped to $a$ under $\Phi$ satisfying $\operatorname{deg}\left(\Phi, z_{n}\right) \geq n$, so $\Phi$ is not strongly subhyperbolic.

Altogether, this means that $\Phi(z)$ is subhyperbolic, $A(\Phi)=\emptyset$, but for every $n \in \mathbb{N}$ there exists a point $z_{n} \in \mathcal{J}(\Phi)$ such that $\operatorname{deg}\left(\Phi, z_{n}\right) \geq n$, yielding the desired example.

Remark. It is not hard to see that the presented construction can be generalized. For more details see Sections 2 and 4 in [13].

\section{ACKNOWLEDGEMENTS}

The author gives special thanks to her Ph.D. supervisor, Lasse Rempe, for introducing her to the research problem and for his continuous help and support. Furthermore, the author would like to thank Adam Epstein and Mary Rees for interesting and helpful discussions. The author is also grateful for the referee's comments that led to clarification of constructions and improvements in the exposition of the article.

\section{REFERENCES}

1. J. M. Aarts and L. G. Oversteegen, 'The geometry of Julia sets', Trans. Amer. Math. Soc. 338, no. 2 (1993), 897-918. MR.1182980 (93j:30021)

2. A. F. Beardon and C. Pommerenke, 'The Poincaré metric of plane domains', J. London Math. Soc. (2) 18, no. 3 (1978), 475-483. MR.518232 (80a:30020)

3. W. Bergweiler, 'Iteration of meromorphic functions', Bull. Amer. Math. Soc. 29, no. 2 (1993), 151-188. MR1216719 (94c:30033)

4. A. Douady and J. Hubbard, 'Etude dynamique des polynômes complexes', Publications Mathèmatique d'Orsay, 84, Université de Paris-Sud (1984). MR762431 (87f:58072a)

5. D. Drasin and Y. Okuyama, 'Singularities of Schröder maps and unhyperbolicity of rational functions', Comput. Methods Funct. Theory 8, no. 1 (2008), 285-302. MR2419479 (2009d:37078)

6. A. E. Eremenko, 'On the iteration of entire functions', Dynamical Systems and Ergodic Theory, Proc. Banach Center Publ. Warsaw 23 (1989), 339-345. MR.1102727 (92c:30027)

7. A. E. Eremenko and M. Y. Lyubich, 'Dynamical properties of some classes of entire functions', Ann. Inst. Fourier, Grenoble 42, no. 4 (1992), 989-1020. MR.1196102 (93k:30034)

8. J. Graczyk and G. Światek, 'The real Fatou conjecture', Annals of Mathematics Studies, Princeton University Press (1998). MR1657075 (2000a:37020)

9. J. K. Langley and J.H. Zheng, 'On the fixpoints, multipliers and value distribution of certain classes of meromorphic functions', Ann. Acad. Sci. Fenn. 23 (1998), 135-150. MR 1601855 (99b:30044)

10. C. T. McMullen, 'Complex dynamics and renormalization', Annals of Mathematics Studies, Princeton University Press 135, 3rd edition (1994). MR1312365 (96b:58097)

11. H. Mihaljević-Brandt, 'A landing theorem for dynamic rays of geometrically finite entire functions', J. London Math. Soc. (2) 81 (2010), 696-714. MR2650792 
12. H. Mihaljević-Brandt, 'Topological Dynamics of Transcendental Entire Functions', Ph.D. Thesis, University of Liverpool, 2009.

13. H. Mihaljević-Brandt and J. Peter, 'Poincaré functions with spiders' webs', to appear in Proceedings of the AMS.

14. J. Milnor, 'Dynamics in one complex variable', Annals of Mathematics Studies, Princeton University Press, Princeton, NJ, Vol. 160, 3rd edition (2006). MR2193309 (2006g:37070)

15. R. Nevanlinna, 'Eindeutige analytische Funktionen', Springer-Verlag/Berlin, Göttingen, Heidelberg, 2nd edition (1953). MR0057330 (15:208c)

16. H. Poincaré, 'Sur une classe nouvelle de transcendantes uniformes', J. Math. Pures Appliquées IV Ser. 6 (1890), 316-365.

17. L. Rempe, 'Topological dynamics of exponential maps on their escaping sets', Ergodic Theory Dynamical Systems 26, no. 6 (2006), 1939-1975. MR2279273 (2008c:37069)

18. L. Rempe, 'Rigidity of escaping dynamics for transcendental functions', Acta Math. 203, no. 2 (2009), 235-267. MR2570071 (2011b:37084)

19. L. Rempe, 'The escaping set of the exponential', Ergodic Theory Dynam. Systems 30, no. 2 (2010), 595-599. MR2599894 (2011h:37069)

20. P. J. Rippon and G. M. Stallard, 'Escaping points of entire functions of small growth', Math. Z. 261, no. 3 (2009), 557-570. MR2471088 (2010a:30043)

21. G. Rottenfußer and D. Schleicher, 'Escaping points of the cosine family', Transcendental dynamics and complex analysis, London Mathematical Society Lecture Notes Series, Cambridge University Press 348 (P. J. Rippon and G. M. Stallard, eds.) (2008). MR2458810(2009j:37069)

22. G. Rottenfusser, J. Rückert, L. Rempe and D. Schleicher, 'Dynamic rays of bounded-type entire functions', Ann. Math. (2) 173, no. 1 (2011), 77-125. MR2753600

23. D. Schleicher and J. Zimmer, 'Periodic points and dynamic rays of exponential maps', Ann. Acad. Sci. Fenn. Math. 28 (2003), 327-354. MR1996442(2004e:37068)

24. D. Schleicher, 'The dynamical fine structure of iterated cosine maps and a dimension paradox', Duke Math. Journal 136, no. 2 (2007), 343-356. MR2286634 (2008d:37078)

25. D. Schleicher, 'Hausdorff dimension, its properties, and its surprises', American Mathematical Monthly 114 (2007), 509-528. MR2321254 (2008d:54024)

26. W. P. Thurston, 'Geometry and topology of three-manifolds', Princeton Lecture Notes (1979).

27. W. P. Thurston, 'On the combinatorics and dynamics of iterated rational maps', Preprint (1984).

28. G. Valiron, 'Fonctions analytiques', Presses Universitaires de France (1954). MR0061658 $(15: 861 \mathrm{a})$

Mathematisches Seminar der Christian-Albrechts-Universität zU Kiel, 24118 Kiel, Germany

E-mail address: helenam@math.uni-kiel.de 